\title{
Un lot de vaisselle moderne déposé dans l'église Sainte-Anne de Boulbon (Bouches-du-Rhône)
}

In: Archéologie du Midi médiéval. Tome 8-9, 1990. pp. 155-168.

\section{Résumé}

Cette étude présente un lot de vaisselle moderne, découvert en 1982 lors de travaux, dans une ancienne sacristie murée de l'église Sainte-Anne de Boulbon, petit village provençal situé aux confins des Bouches-du-Rhône et du Vau- cluse. Le matériel abandonné (220 pièces) illustre le rôle de sociabilité qui fut celui de l'église au sein d'une communauté villageoise. II permet de confirmer le répertoire des formes utilisées au XVIle siècle en Provence. Les plus nombreuses en pâte calcaire sont issues d'ateliers proches (Avignon ? Tarascon ?...) et celles en pâte kaolinitique réfractaire originaires des célèbres ateliers de l'Uzège. La présence dans ce milieu clos, d'une faïence importée de Ligurie du début du XVIle siècle, renforce la chronologie proposée par l'ensemble du vaisselier régional et par le prix-fait de l'église qui fixe un terminus aquo.

\section{Abstract}

The purpose of this paper is the presentation of a Modern group of ceramics found during repair works in 1982 in the ancient walled up sacristy of the church Sainte-Anne in Boulbon, a small village in Provence located on the frontier of the Bouches-duRhône and the Vaucluse districts. This material (220 pièces) reflects the role of sociability of the church within the villager's community. It provides evidence of the repertoire of forms of the 17th century in Provence. For the most part in calcareous clay, these ceramics were produced in the workshops in the vicinity (either Avignon or Tarascon) and the ones in kaolinic clay are originated in the workshops of Uzège.

The presence in this closed context, of a faience imported from Liguria dated at the beginning of the 17th century rein orces the chronology suggested by the regional « dresser» on the one hand the prix-fait of the church on the other hand which both they established a terminus aquo.

Citer ce document / Cite this document :

Betton Félicien, Buravand Véronique, Vallauri Lucy. Un lot de vaisselle moderne déposé dans l'église Sainte-Anne de Boulbon (Bouches-du-Rhône). In: Archéologie du Midi médiéval. Tome 8-9, 1990. pp. 155-168.

doi : 10.3406/amime.1990.1208

http://www.persee.fr/web/revues/home/prescript/article/amime_0758-7708_1990_num_8_1_1208

\section{(3) $\Theta \Theta$}




\title{
UN LOT DE VAISSELLE MODERNE, DEPOSE DANS LEGLISE SAINTE-ANNE DE BOULBON
}

\author{
(Bouches-du-Rhône) \\ Félicien BETTON, Veronique BURAVAND, Lucy VALLAURI (*)
}

\begin{abstract}
Cette étude présente un lot de vaisselle moderne, découvert en 1982 lors de travaux, dans une ancienne sacristie murée de l'église Sainte- $\Lambda$ nne de Boulbon, petit villagc provençal situé aux confins des Bouches-du-Rhône et du Vaucluse. Le matériel abandonné ( 220 pièces) illustre le rôle de sociabilité qui fut celui de l'église au sein d'une communauté villageoise. Il permet de confirmer le répertoire des formes utilisées au XVII ${ }^{\mathrm{c}}$ siècle en Provence. Les plus nombreuses en pâte calcaire sont issues d'ateliers proches (Avignon? Tarascon?...) et celles en pâte kaolinitique réfractaire originaires des célèbres ateliers de l'Uzège.

La présence dans ce milieu clos, d'une faïence importée de Ligurie du début du XVII siècle, renforce la chronologie proposée par l'ensemble du vaisselier régional et par le prix-fait de l'église qui fixe un terminus aquo.
\end{abstract}

The purpose of this paper is the presentation of a Modern group of ceramics found during repair works in 1982 in the ancient walled up sacristy of the church Sainte-Anne in Boulbon, a small village in Provence located on the frontier of the Bouches-du-Rhône and the Vaucluse districts. This material (220 pieces) reflects the role of sociability of the church within the villager's community. It provides evidence of the repertoire of forms of the 17th century in Provence. For the most part in calcareous clay, these ceramics were produced in the workshops in the vicinity (either Avignon or Tarascon) and the ones in kaolinic clay are originated in the workshops of Uzège.

The presence in this closed context, of a faience imported from Liguria dated at the beginning of the 17th century reinforces the chronology suggested by the regional "dresser» on the one hand the prix-fait of the church on the other hand which both they established a terminus aquo.

C'est à l'occasion de travaux de réfection de l'église Sainte-Anne qu'un important lot de vaisselle moderne a été découvert en 1982, dans le village de Boulbon situé aux confins des Bouches-du-Rhône, à $5 \mathrm{~km}$ de Tarascon et $18 \mathrm{~km}$ d'Avignon (Vallauri 1989: 49).

220 pièces encore empilées les unes sur les autres ont été exhumées de façon fortuite dans un réduit voûté adjacent au chœur qui supportait l'escalier menant au clocher, et dont les ouvertures avaient été murées. On accédait à cet espace depuis l'édifice, mais une porte communiquait également avec l'extérieur au niveau de la rue. La présence d'une piscine dans la pièce permet de penser qu'il s'agissait d'une minuscule sacristie de l'église dont la reconstruction à partir de 1626 est connue par un prix-fait.
Dès 1621 , les consuls achètent la vieille chapelle Saint-Sébastien « laquelle s'en va en ruine, en danger de perdre la futaille». On construira la nouvelle église sur son emplacement. En juillet 1626, le bail du prifait est donné à Guillaume Babeau, maçon. L'acte du prifait de la nouvelle église passé devant Maître Jean Bourg, notaire à Boulbon, le 31 juillet 1626 est très détaillé. L'église aura 11 cannes de long, 4 cannes de large et 5 de haut. La canne de Boulbon étant de $2 \mathrm{~m}$, on aura $22 \mathrm{~m}$ sur $8 \mathrm{~m}$ et $10 \mathrm{~m}$ de haut. Mention est faite de quelques travaux effectués par la suite. En 1629, un maçon du village, François Notton, touche pour un travail de «petite réparation» la somme d'l livre 6 sols. Le clocher n'est fini de bâtir qu'en 1680 (Arch. Com. de Boulbon. BB 10, DD 19 , pièce $\mathrm{n}^{\circ} 29$ ). 
La sacristie trop exiguë fut sans doute condamnée à la suite des agrandissements latéraux et de la création d'un nouveau local à l'ouest que nous pouvons encore observer aujourd'hui (Fig. 1).

Les céramiques présentées ici ne peuvent être replacées dans un contexte archéologique, aucune observation n'ayant été faite au moment de la découverte. Le matériel trouvé sans enfouissement a cependant été soigneusement prélevé par les inventeurs. Il est de plus issu d'un millieu clos, et est à première vue parfaitement homogène.

Son étude exhaustive s'inscrit dans le cadre des recherches sur la céramique post-médiévale en Provence, connue essentiellement par des contextes de fouilles de sites consommateurs avignonnais, marseillais ou varois (Démians d'Archimbaud $1980 \mathrm{~A}$, Foy 1986, Carrazé 1987, Abel 1987-88, Pasqualini 1988, Carru 1989).

Cette série de céramiques peut donc être resituée
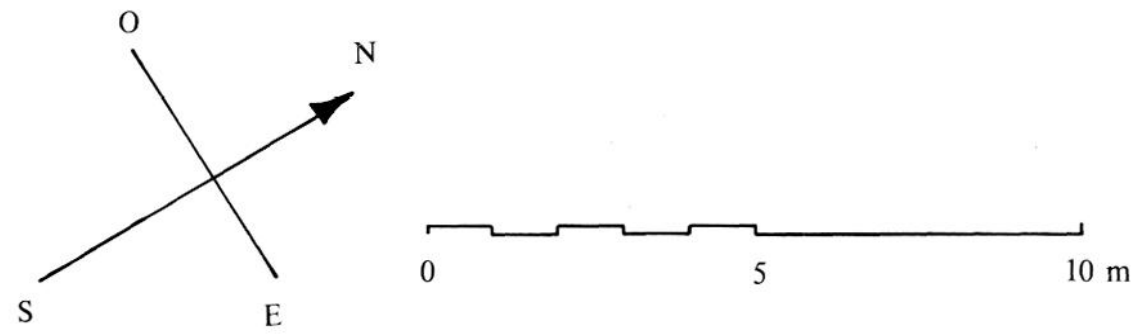

dans une chronologie relative. De plus, elle s'intègre dans la détermination des sites producteurs de mieux en mieux cernés par les études menées à partir des textes (Amouric 1986), des recherches sur le terrain (Thiriot $1985 \mathrm{~A}$ et B) et des analyses géochimiques réalisées au Laboratoire de céramologie de Lyon par M. Picon (ERA 3 du CRA). Toutes ces méthodes conjointes sont menées sur la longue durée.

Les principaux résultats obtenus ont permis de reconnaître que deux catégories d'argile ont été employées de façon privilégiée dans la basse vallée du Rhône : l'argile calcaire beige-rouge "banale", réservée principalement à la vaisselle de table, et la pâte kaolinitique en particulier celle de l'Uzège : blanche violacée feuilletée, aux qualités réfractaires, mieux adaptée à la fabrication des vases culinaires (Démians d'Archimbaud B : 15-28). Cette dualité est un phénomène permanent qui a été observé au Moyen Age, à l'époque moderne et contemporaine, à partir de toutes les études céramologiques provençales et languedociennes.

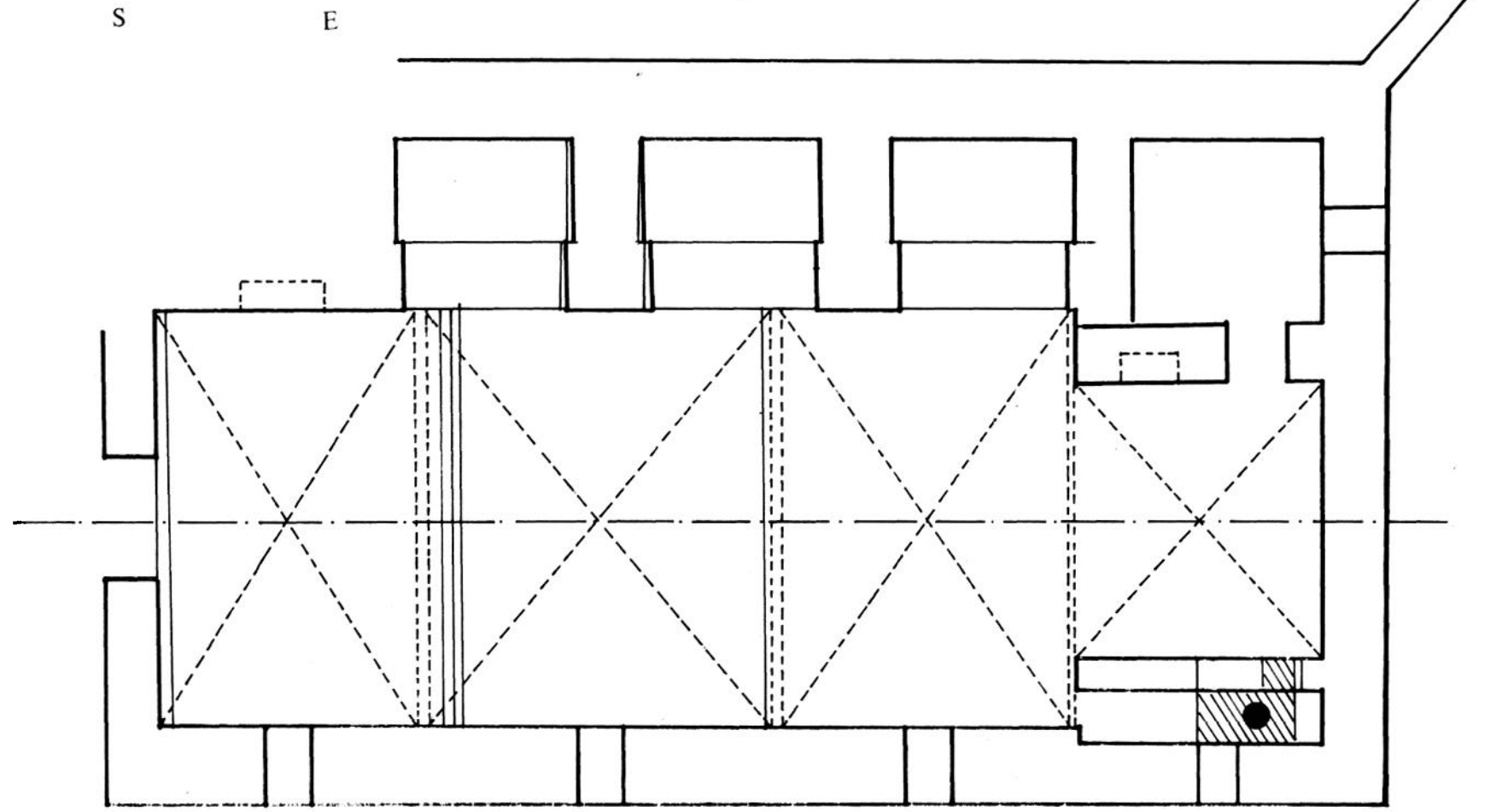

Fig. 1: Plan de l'église Sainte Anne. Localisation de la découverte, d'après M. Dufoy, Architecte en Chef des Monuments Historiques.

\section{UNE FAǏENCE D'IMPORTATION LIGURE}

Parmi ces céramiques d'origine régionale, toutes produites selon la même technique d'argile engobée puis recouverte de glaçure plombifère, une pièce est unique à tous points de vue tant par sa pâte blanche de texture sableuse que par son revêtement d'émail stannifère opaque et son origine (Fig. 2). Il s'agit d'un vase sur pied d'importation ligure orné d'une applique en relief en forme de masque de lion et d'un décor peint au bleu de cobalt. La forme est incomplète et le détail de l'ouverture et du pied inconnu. Cependant le profil général reste dans la tradition des hydries renaissantes où la bouche du masque sert de robinet (Barile 1975). Sur la panse du vase se développe un décor de deux oiseaux à longue queue, figés au milieu d'une végétation très dense, composée de feuilles allongées de palmettes et de fleurs encadrant le masque. Sur le pied et le registre inférieur de la panse court une frise géométrique, où alternent des traits horizontaux, verticaux et des points alignés. Sous le pied, au revers les points disposés en rosaces ne sont pas sans rappeler une marque de fabrique qui orne fréquemment l'extérieur des faïences modernes. L'ensemble de ce décor qui caractérise le 
style calligraphique naturaliste d'Albisola en Ligurie, puise son inspiration dans le répertoire oriental, islamique et chinois (Marzinot 1979). Ce style en faveur au début du XVII ${ }^{c}$ siècle, se retrouve sur toutes les hydries aux formes encore légères et harmonieuses. La présence de cette faïence exceptionnelle d'importation constitue un premier jalon chronologique pour dater l'ensemble des céramiques communes dont l'étude détaillée suit.

2. LES CERAMIQUES REGIONALES : PREDOMINANCE DES PATES CALCAIRES ET DES FORMES OUVERTES (Tableau 1)

La vaisselle en pâte kaolinitique de l'Uzège est nettement minoritaire puisque sur un total de 220 objets, elle ne représente que $19 \%$ de l'ensemble des écuelles et vases. Elle est dans tous les cas monochrome et sans décor.

La vaisselle en pâte calcaire qui domine est également majoritairement monochrome, mais dans 32\% des cas, elle comporte des décors polychromes d'engobes.

Le répertoire des formes est quasiment identique, pour les deux pâtes utilisées. Les $3 / 4$ des vaisselles sont des formes ouvertes: la première forme est l'écuelle (184 - forme A), suivie par l'assiette à marli (19 - forme $\mathrm{B})$, puis quelques pots à cuire de type pégau ou toupin, quelques vases à liquides et une faisselle.

Cette constatation faite à partir du lot de Boulbon renforce l'idée de la prépondérance de l'écuelle à l'épo- que moderne, en Provence. Cette forme de portion individuelle prédomine dans tous les contextes archéologique étudiés du XVI ${ }^{\mathrm{e}}$ et $\mathrm{XVII} \mathrm{I}^{\mathrm{e}}$ siècle. Au siècle suivant, elle sera concurrencée et remplacée par l'assiette à marli (Foy 1986).
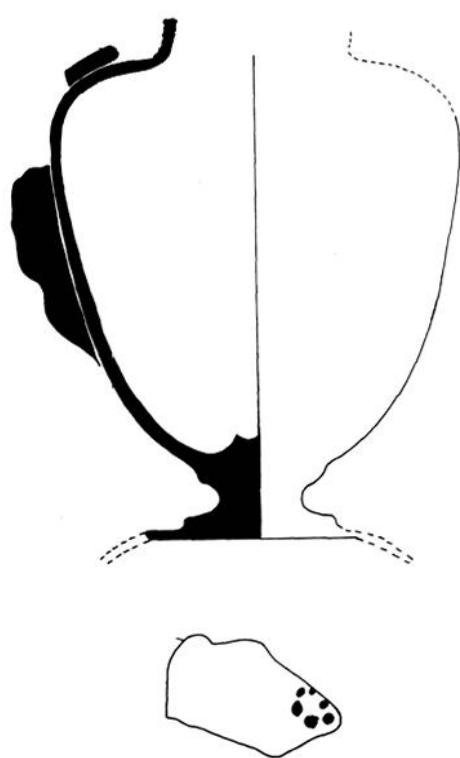

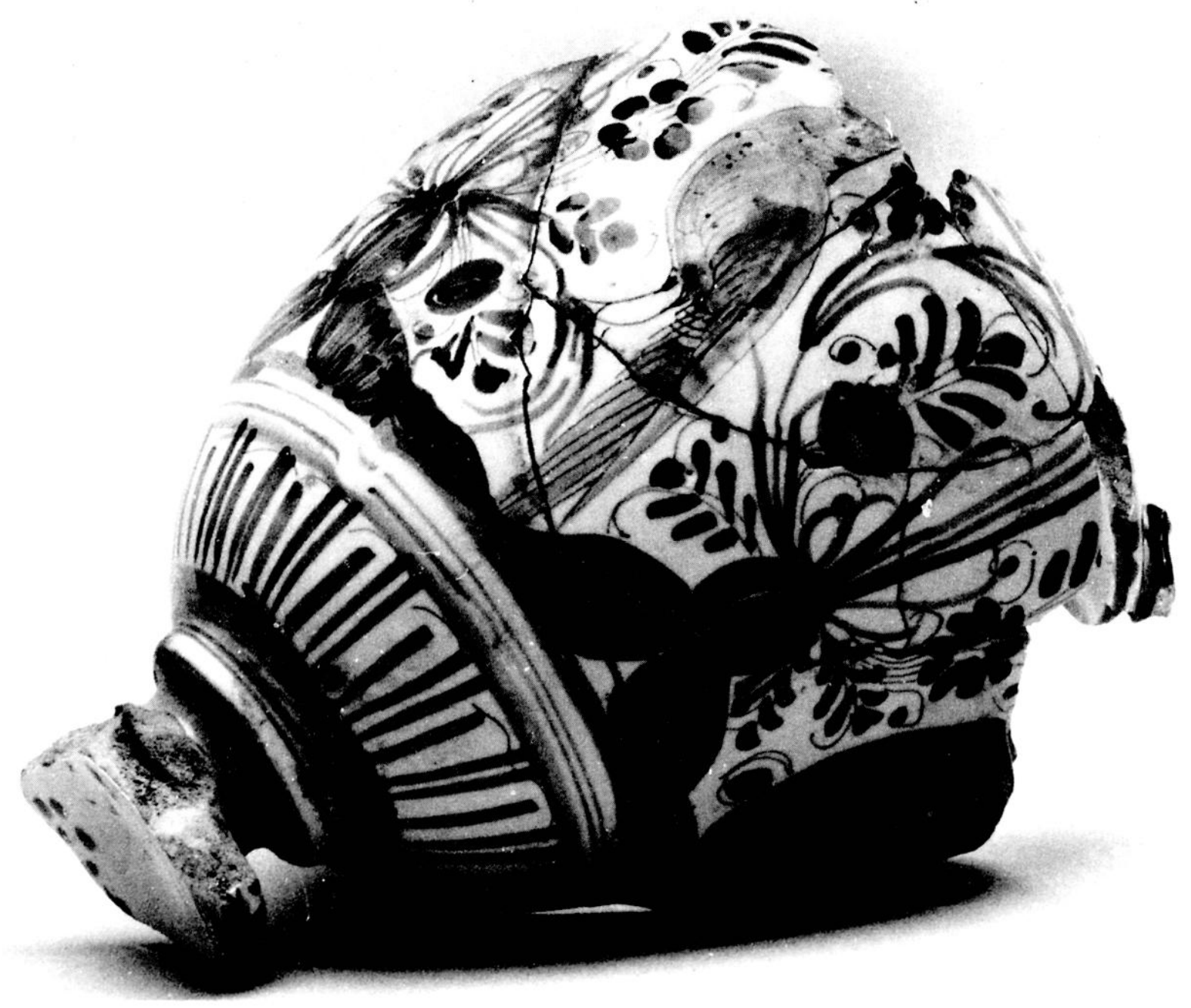

Fig. 2: Hydrie à décor bleu : faïence d’origine ligure, dessin éch. 1/3, photo éch. $1 / 1$ (V. Buravand). 


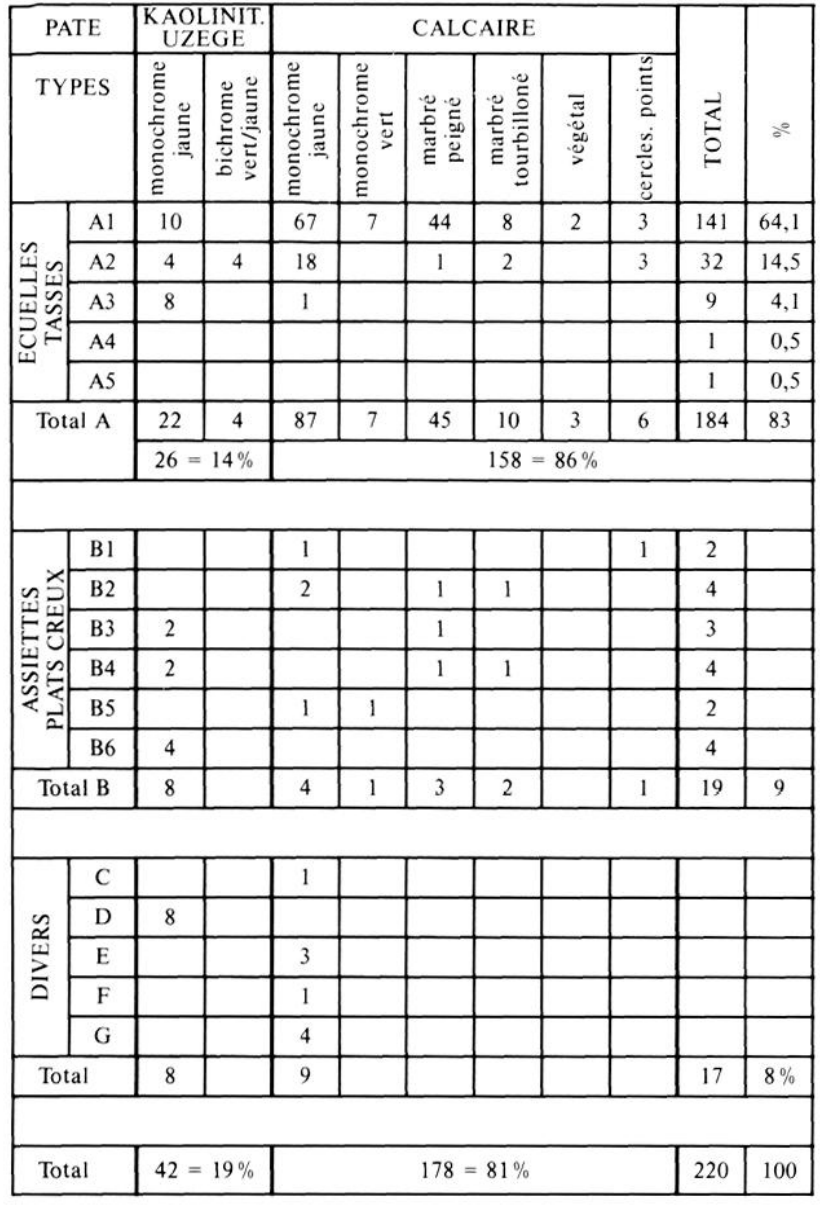

Tableau 1: Comptage de l'ensemble de la vaisselle par pâte et type.

\subsection{Typologie des céramiques en pâte engobée et} glaçurée (Tableau 1)

\subsubsection{Les formes ouvertes}

Elle prédominent puisqu'on dénombre dix types (contre cinq types de formes fermées) et parmi elles les écuelles sont les plus nombreuses : 184 , soit plus de $83 \%$ du total des céramiques.

A l'intérieur de ce groupe, on peut distinguer quatre catégories morphologiques différentes :

- les écuelles hémisphériques, A1-A3;

- les écuelles à fond plat, $\mathrm{A} 2$;

- les assiettes, B1-B2-B3-B4-B5;

- les plats creux, B6.

2.1.1.1. Les écuelles hémisphériques (Types A1-A3) sont de loin les plus représentées: on en compte 150, c'est-à-dire plus de $80 \%$ du lot d'écuelles. Elles possèdent un fond concave, des parois plus ou moins évasées devenant assez souvent rentrantes. Selon les pièces, la hauteur varie entre $4 \mathrm{~cm}$ et $6 \mathrm{~cm}$ et le diamètre d'ouverture entre $10,5 \mathrm{~cm}$ et $14,5 \mathrm{~cm}$ (Fig. $3: 1$ à 5). Quant au pied, il peut être plus ou moins marqué (la hauteur maximale est de $9 \mathrm{~mm}$ ) voire pas du tout, et le diamètre compris entre $4,5 \mathrm{~cm}$ et $6 \mathrm{~cm}$. Parmi ces écuelles, la majorité (141 sur 150) possède des oreilles alors que les autres n'ont pas de moyen de préhension mais présente une carène pour une d'entre elles (Fig. $3: 4$ ) et une rupture de panse à peine marquée pour les autres. Seul ce bol fortement caréné est engobé et vernis à l'intérieur et à l'extérieur alors que pour le reste du groupe des écuelles hémisphériques, l'engobe et le vernis ne recouvrent que l'intérieur.
La carène n'est pas réservée aux écuelles sans préhension, puisque quatre d'entre elles présentant des oreilles en possèdent une très nette. Ces 4 écuelles (Fig. $3: 5$ ) sont absolument identiques par paire, aussi bien par leurs dimensions que par leur couleur et leur décor. Il est en effet à noter que les bols sans préhension sont tous monochromes à la différence des autres qui peuvent être aussi bien monochromes que décorés.

Ce type de forme hémisphérique est présent parmi les céramiques à pâte calcaire aussi bien que parmi celles à pâte kaolinitique de l'Uzège.

2.1.1.2. Type A2: Il en est de même pour les écuelles à fond plat ( 24 en pâte calcaire et 8 en pâte kaolinitique) qui forment le deuxième groupe morphologique, loin derrière les écuelles hémisphériques : $17 \%$ du total des écuelles. Leurs parois sont rectilignes ou légèrement évasées et leur fond est large (entre $10 \mathrm{~cm}$ et $12 \mathrm{~cm}$ ) et plat. Elles ont un diamètre d'ouverture compris entre 13 et $16,5 \mathrm{~cm}$ et une hauteur de $5 \mathrm{~cm}$ environ (Fig. $3: 6-7$ ). Certaines d'entre elles (et en particulier celles en pâte kaolinitique) sont recouvertes d'un engobe et d'un vernis à l'intérieur comme à l'extérieur, mais pour la majorité, la pâte apparaît nue sur la face extérieure. Cette forme est le plus souvent monochrome, mais dans quelques cas, décorée.

A l'inverse des écuelles hémisphériques, celles à fond plat possèdent toutes des oreilles moulées, "tréflées" ou "géométriques".

- Les oreilles : une étude plus précise des oreilles s'impose ici dans la mesure où celles-ci ont des formes variées. De plus, les écuelles à oreilles étant les plus nombreuses dans ce lot, il apparaît intéressant de faire un essai de classification : tel type d'oreille est-il spécifique d'une forme particulière d'écuelle? (Tableau 2). Parmis les nombreuses variations, trois groupes d'oreilles peuvent être distingués, et tout d'abord les oreilles moulées (Fig. 5 : 10). Elle sont présentes sur 95 écuelles dont 79 sont de forme hémisphérique et 16 à fond plat. De plus, sur les 79 écuelles de type Al, 50 sont monochromes et 29 décorées, et parmi les 16 de type A2, 13 sont monochromes et 3 décorées. Certaines semblent assez sommaires, alors que d'autres sont plus raffinées et représentent par exemple, une fleur de lys. On reconnaît environ dix modèles d'oreilles moulées dont un est réservé aux écuelles en pâte de l'Uzège (Fig. $5: 7$ ), alors que certains autres figurent indifféremment sur les écuelles à pâte calcaire ou kaolinitique.

En revanche, les oreilles modelées de forme «tréflée » ou « pincée » n'existent que sur les bols à pâte calcaire. Elles évoquent en effet un trèfle dont l'extrémité des feuilles serait plus ou moins pointue (Fig. 5:9). Leur exécution semble rapide et le résulat dans l'ensemble est assez grossier. 44 écuelles de type Al ont des oreilles «tréflées" contre 12 de type A2.

La troisième sorte d'oreilles modelées que nous appelerons «géométriques » est beaucoup moins représentée : elle ne concerne que 13 écuelles (12 hémisphériques et 1 à fond plat). Il s'agit de la forme la plus simple (Tableau 2), ou du moins la plus géométrique puisqu'elle n'est en fait qu'un carré aux angles plus ou moins émoussés, voire un rectangle (au point de rattachement avec la paroi) accolé à un carré.

Hormis le fait que les oreilles moulées sont les plus nombreuses et que parmi elles, un genre précis est réservé aux écuelles de type A 2 en pâte de l'Uzège, on ne peut pas dire que, proportionnellement, une sorte précise d'oreilles soit propre à une forme ou un décor particulier.

2.1.1.3. Les pièces uniques: il n'existe qu'une seule tasse (Type A4) (Fig. 4:1) dans le lot de céramiques de Boulbon. Elle est de petites dimensions et possède une 

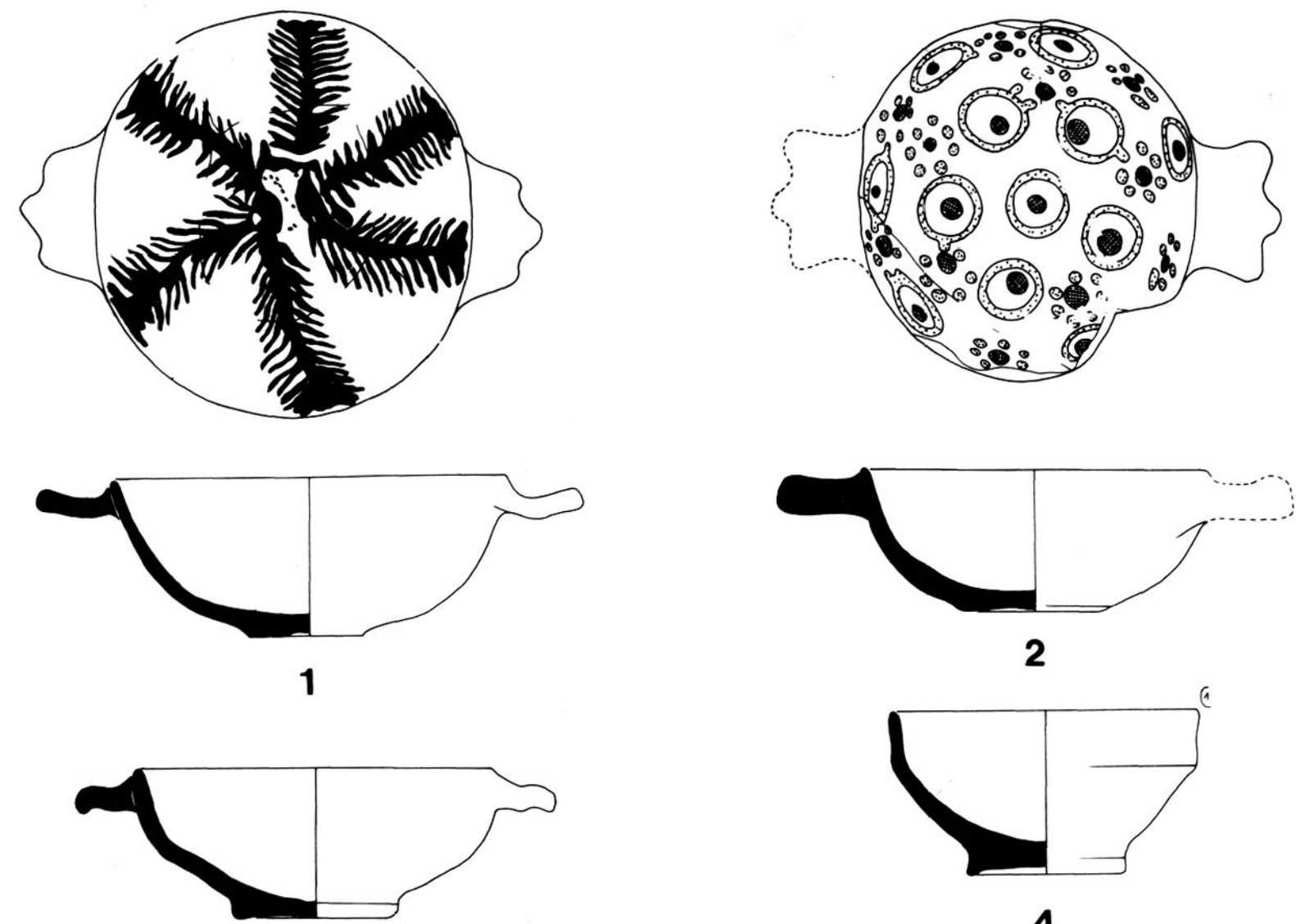

3
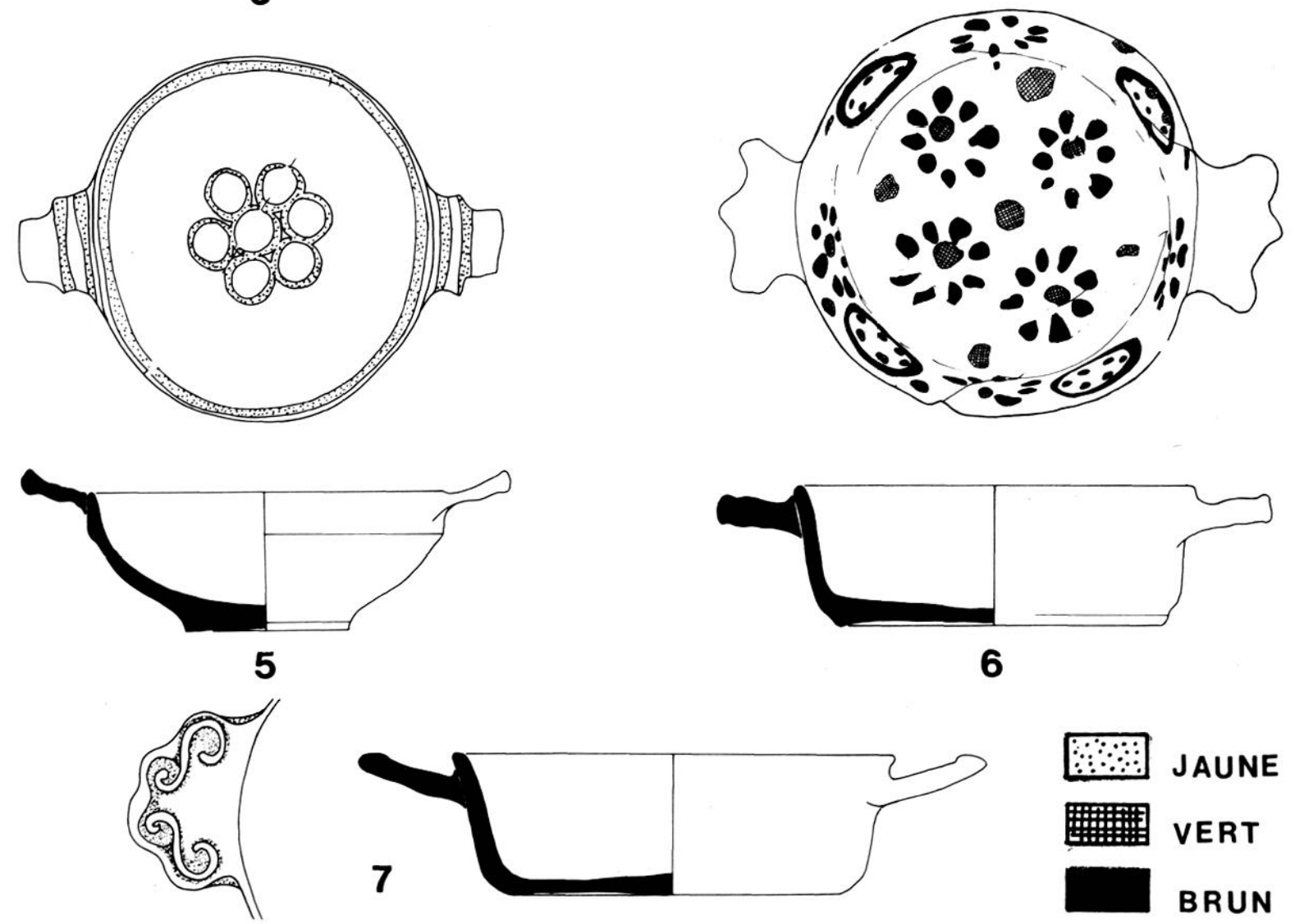

Fig. 3 : Ecuelles: 1-2-3-5, type A1;4, type A3; 6-7, type A2. 


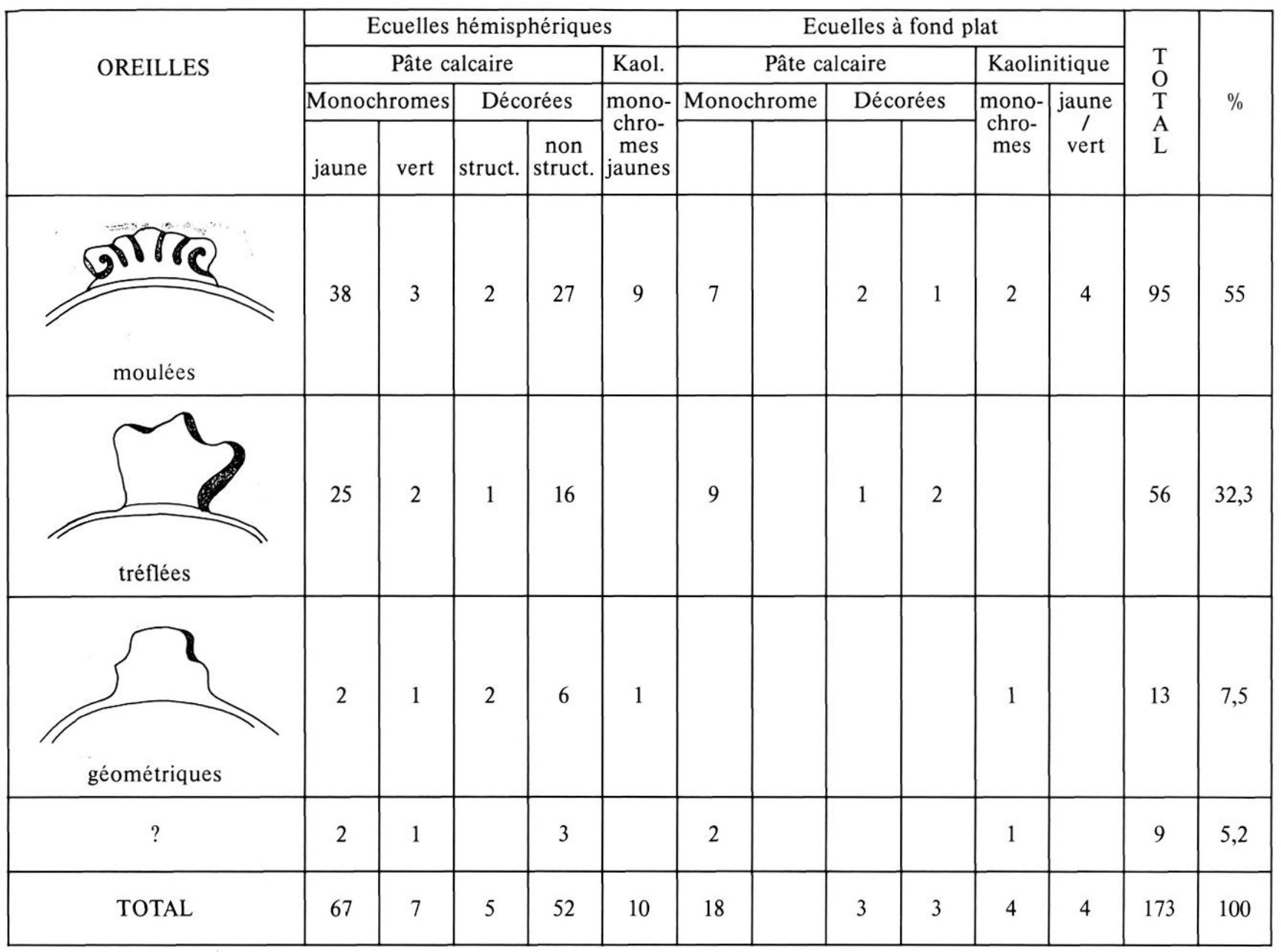

Tableau 2: typologie des oreilles de préhension. Les écuelles.

anse assez verticale. Son pied est bien marqué, le fond est concave et les parois évasées jusqu'au niveau d'une carène; elles sont ensuite rectilignes voire rentrantes. La forme simple et élégante est en pâte calcaire, glaçurée et décorée à l'engobe.

L'ensemble des bols ou écuelles comprend un autre type rare en pâte calcaire, qui est lui aussi représenté par une pièce unique : il s'agit d'un bol caréné (Type A5) de forme exceptionnelle. Son pied est net, les parois s'ouvrent jusqu'au niveau de la carène, puis continuent à s'évaser mais de façon moins brutale. Le diamètre d'ouverture $(15,7 \mathrm{~cm}$ ) est important (Fig. $4: 2)$. Ce bol possède deux anses verticales rattachées au corps de l'objet sous la carène. Il est recouvert d'un engobe et d'une glaçure à l'intérieur avec quelques coulures à l'extérieur.

Dans la typologie des formes ouvertes, une faisselle (Type C) occupe une place particulière par sa fonction spécifique (Fig. 4:3). Le fond ( $9 \mathrm{~cm}$ de diamètre) est percé de cinq trous permettant de laisser s'égoutter le fromage. Cette forme fonctionnelle est tournée dans une argile calcaire.

2.1.1.4. Les assiettes (Types B1 à B5). Il existe 2 petites assiettes plates à marli $(\mathrm{B} 1)$ : leur diamètre d'ouverture est en moyenne de $16 \mathrm{~cm}$ pour une hauteur de 3 $\mathrm{cm}$ (Fig. 4:4). Le fond est plat, les parois largement évasées et le bord arrondi, tout comme les grandes assiettes plates (B2) dont la seule différence réside dans les dimensions. On en compte 4 , soit $1,8 \%$ du total des céramiques. Ces deux types B1 et B2 sont essentiellement en pâte calcaire, engobée et recouverte d'une glaçure monochrome décorée.
Plusieurs types d'assiettes creuses sont à distinguer, et tout d'abord les petites assiettes creuses (B3) dont deux sont monochromes, en pâte kaolinitique et une décorée, en pâte calcaire. Leur morphologie est comparable à celle des grandes assiettes creuses (B4) (Fig. 4 : 5). Hormis le fait que leur taille est plus réduite, ces types B3 et B4 possèdent un fond concave et un marli dont la largeur varie en $3 \mathrm{~cm}$ et $3,5 \mathrm{~cm}$. Le type $\mathrm{B} 4$ est présent en pâte de l'Uzège pour deux assiettes (monochromes) et en pâte rouge calcaire pour deux autres (décorées).

En revanche, les deux assiettes creuses à marli en pâte calcaire, ont une forme tout à fait originale (Fig. 4 : 6-7). Elle reposent sur un pied de $8 \mathrm{~cm}$ de diamètre, la hauteur est de $5 \mathrm{~cm}$ environ et le diamètre d'ouverture de $23 \mathrm{~cm}$. Pourtant, leur capacité est restreinte par la présence d'un très large marli de $5,5 \mathrm{~cm}$ environ.

Ces deux assiettes, tout comme les autres formes ouvertes étudiées jusqu'à présent, semblent encore destinées à recevoir une portion individuelle, mais sont une forme de transition avec les plats de service creux.

2.1.1.5. Les plats creux (Type B6) : trois plats creux (Fig. $4: 8$ ) semblent plûtot avoir un usage collectif(Thiriot 1983) : ils possèdent en effet un large diamètre d'ouverture, un fond plat pour deux d'entre eux et concave pour le troisième, et un marli d'environ $3,5 \mathrm{~cm}$. Ils sont en pâte kaolinitique et leurs fonds noircis au feu indiquent qu'ils étaient parfaitement adaptés à réchauffer les aliments sur les réchauds produits dans le même centre languedocien (Démians d'Archimbaud 1980, Fig. 56-7-11; Fig. 54-14-15). 

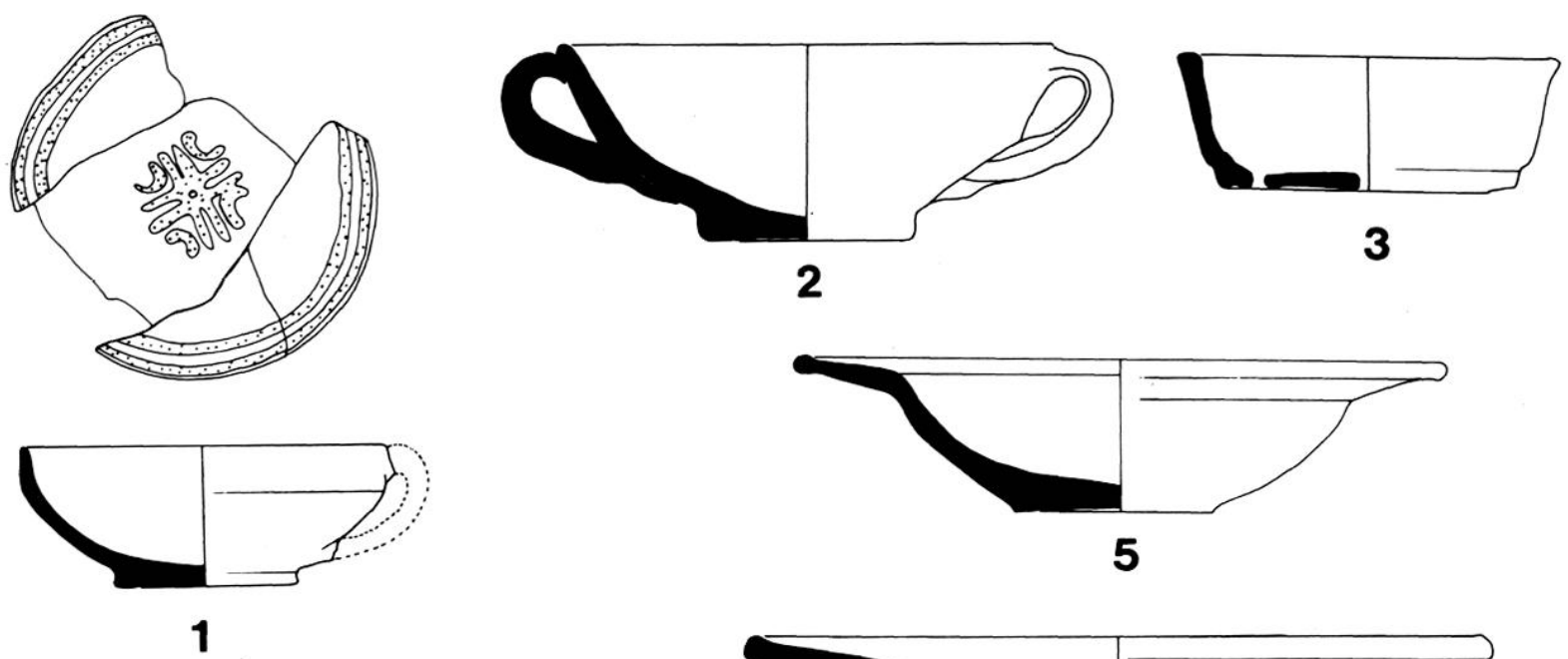

5
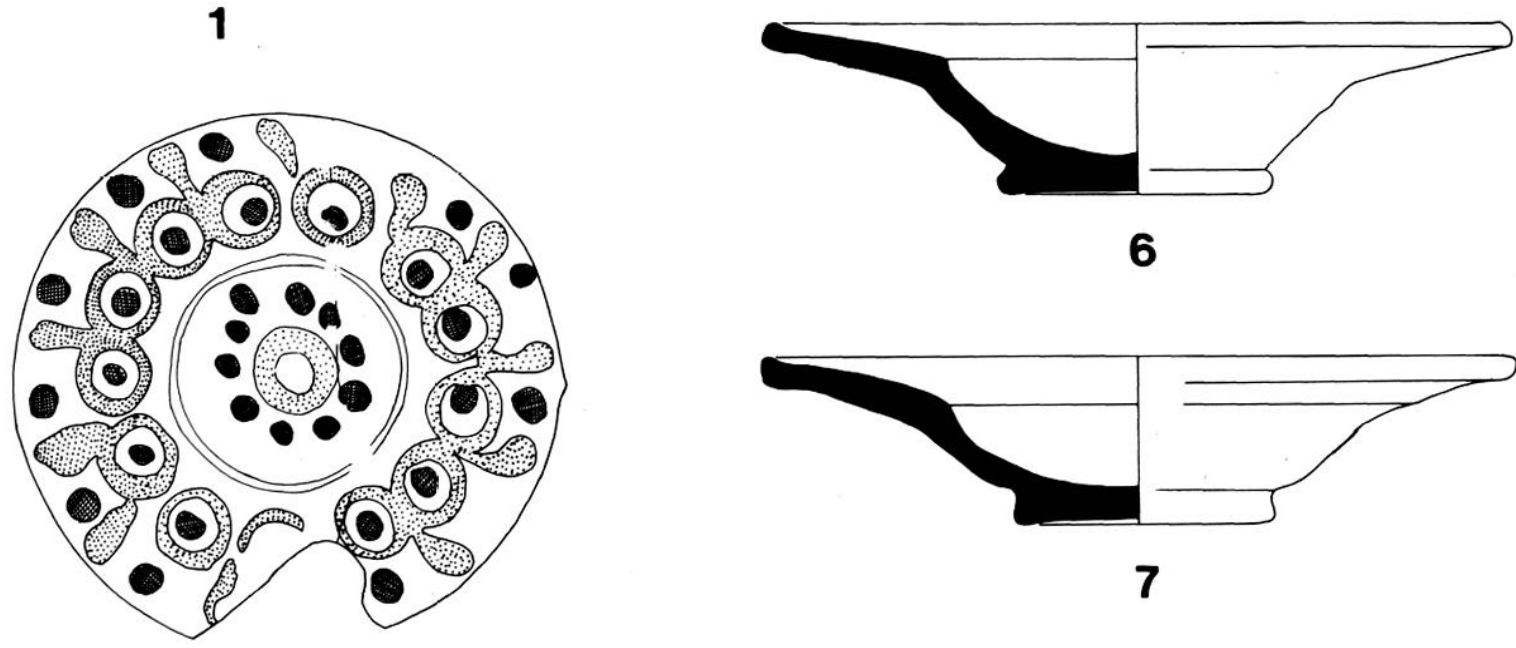

6

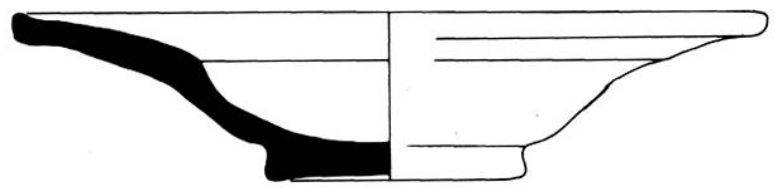

7

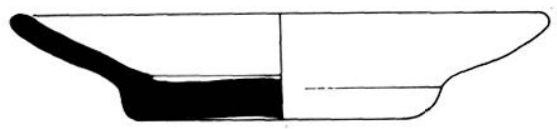

4

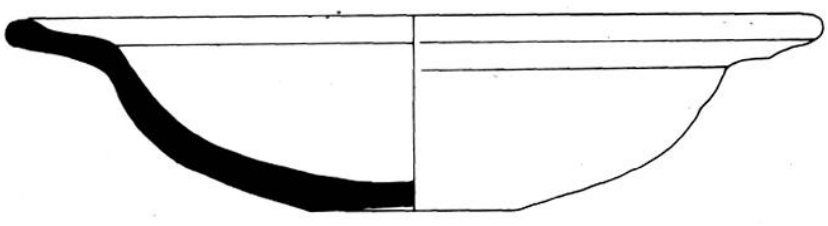

8

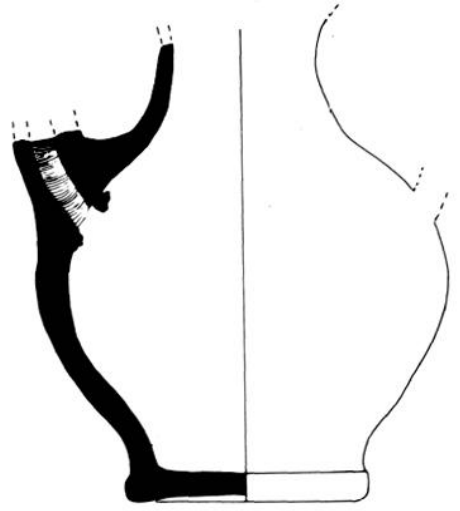

9

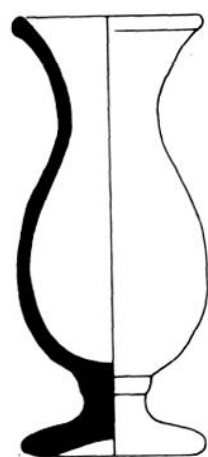

10

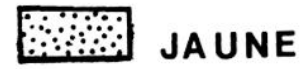

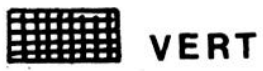

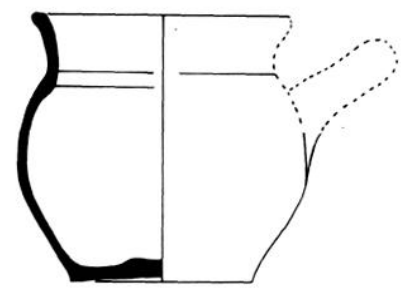

11

Fig. 4 : Tasses : 1-2, types A4 et A5 ; Faisselle : 3, type C; Assiettes : 4, type B1 ; $5:$ type B3, $6:$ type B5, $7:$ type B4, $8:$ type B6 ; Gargoulette: 9 , type F, Vase : 10 , type $G$; Toupin : 11, type E. 


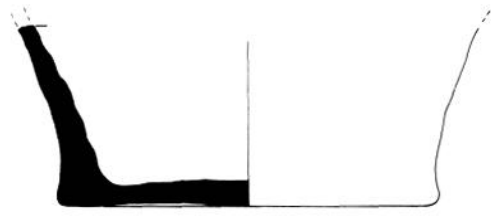

1

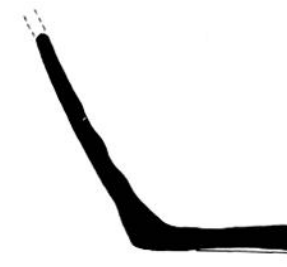

2

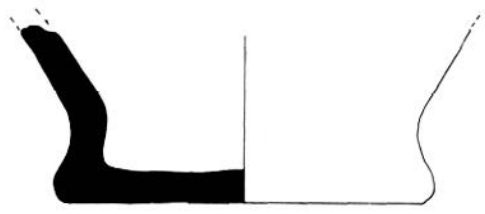

3

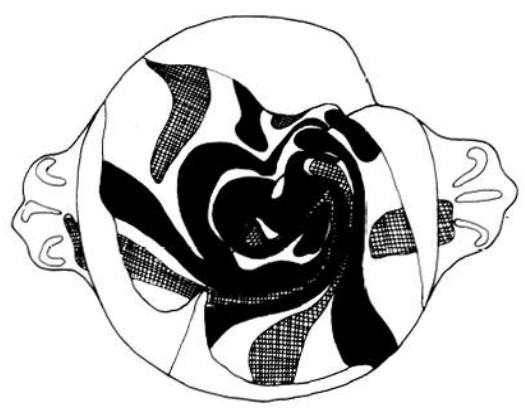

6

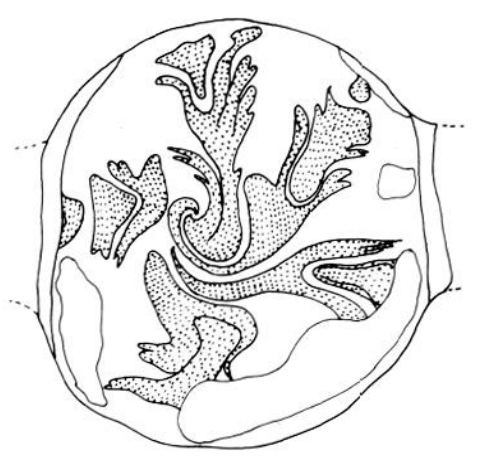

8

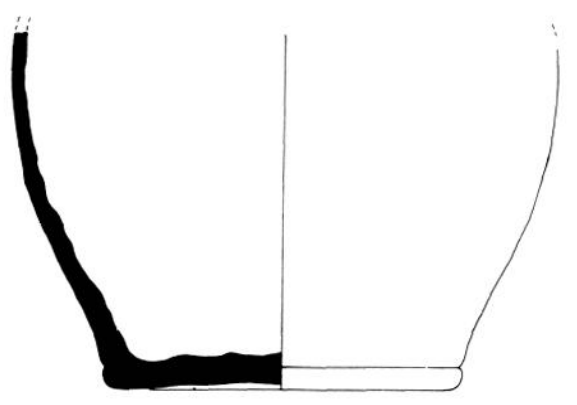

4

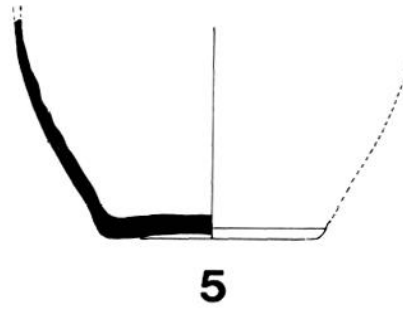

5

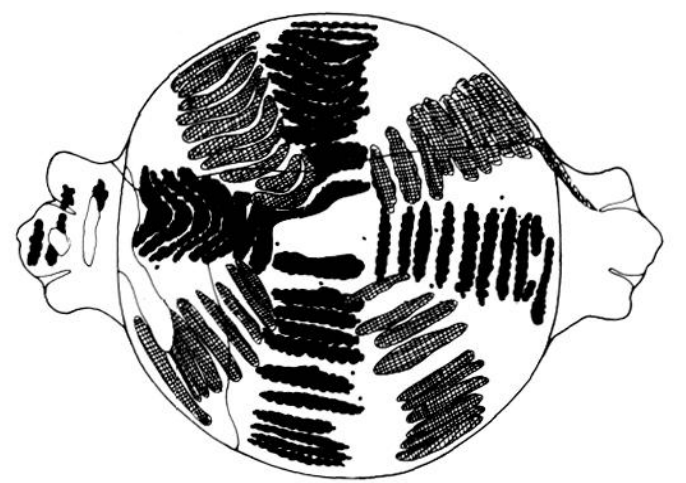

JAUNE 曹册 VERT
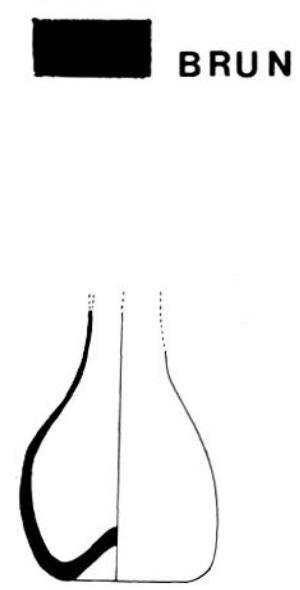

11

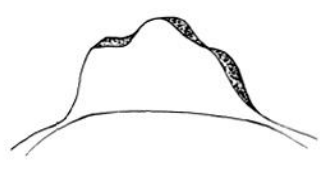

9

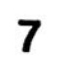

7
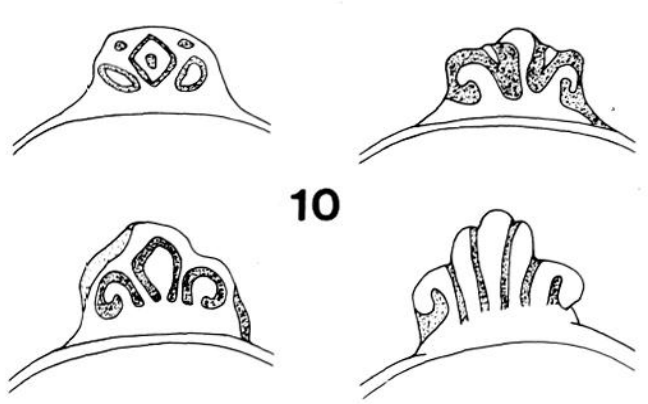

Fig. 5 : Fonds de «pégau» et cruches 1 à $5:$ type $\mathrm{D} ; 6,7,8:$ exemples de décor marbré et végétal $; 9,10$ : oreilles moulées et tréflées. 


\subsubsection{Les formes fermées}

Les pièces entrant dans la catégorie des formes fermées (un peu plus de $7 \%$ du total des céramiques) sont des poteries culinaires et des récipients à liquides: vases, fonds de cruches et gargoulette. Celle-ci (en pâte calcaire) est de forme globulaire et possède un bec verseur tubulaire (Type F). Une anse de préhension semble être rattachée au haut de la panse et à l'extrémité du col (Fig. 4:9).

Les fonds de cruches (Type D) au nombre de trois sont en pâte calcaire (Fig. 5:3) recouverte d'un engobe et d'un vernis à l'intérieur seulement, avec quelques coulures sur l'extérieur des parois. Un rebord de cruche est également présent dans ce lot.

Les quatre vases (en pâte calcaire) (Type G), semblent avoir une fonction décorative. Tous identiques ils ont des petites dimensions ( $13,5 \mathrm{~cm}$ de hauteur), reposent sur un petit pied tronconique et ne possèdent pas d'anse. Leur forme est assez élancée: une panse peu ventrue, un col étroit et une ouverture évasée, symétrique par rapport au pied et terminée par un rebord simple (Fig. 4: 10). Ils sont recouverts d'un engobe rouge et d'unc glaçure à l'intérieur et à l'extérieur.

Quant aux céramiques culinaires (Type D) en pâte de l'Uzège, aux qualités réfractaires nécessaires, de type "pégau» ou toupin, il n'en subsiste que le fond pour sept d'entre elles (Fig. $5: 1-2-4-5$ ). Une seule est entière et de petites dimensions (Fig. 4:11) (Type E). Elle est plus large que haute, possède une panse ventrue, un fond plat et un rebord plat également. Elle était munie d'un manche tubulaire, placé à l'opposé de la paroi noircie par le feu et oblique et comme huit fonds, recouverte à l'intérieur d'un engobe et d'une glaçure.

\subsection{Traitement de surface et techniques décoratives} Cette vaisselle régionale est dans tous les cas recou- verte d'engobe et d'une glaçure plombifère, technique qui en Provence est caractéristique de l'époque moderne. Son apparition se situe à l'extrême fin du Moyen Age et correspond à l'emploi plus généralisé des argiles calcaires pour la vaisselle de table et du goût des techniques décoratives transmises par l'Italie du Nord, plus particulièrement par l'émigration massive des ouvriers ligures dans nos régions (Amouric 1986).

\subsubsection{Les céramiques engobées et recouvertes d'une} glaçure monochrome

Elles sont majoritaires: $68 \%$ qui se répartissent pour la moitié en pâte calcaire et $19 \%$ en pâte kaolinitique de l'Uzège.

La poterie de l'Uzège comme nous l'avons signalé précédemment ne possède à cette époque aucun décor. L'introduction de l'engobe, d'après les fouilles d'ateliers (Thiriot $1985 \mathrm{~A}$ : 43-44) se situerait à partir du XVI ${ }^{\circ}$ siècle. L'emploi de cette couverte blanche de terre unifie la surface de la pâte qui, au Moyen Age, était très blanche, au contraire de l'époque moderne où la majorité des produits sont réalisés dans une argile rose violacée de moins en moins pure. Le vernis plombifère transparent est jaune pâle, miel à jaune verdâtre. La seule fantaisie est un effet de bichromoie obtenu par l'adjonction de cuivre (vert) à l'extérieur de quatre écuelles de types A2 aux oreilles moulées (Fig. 3/7).

Ces écuelles sont en tous points comparables à celles découvertes dans les rebuts de fabrication du four 463 A de Saint-Quentin-la-Poterie (Thiriot 1985 B).

Les céramiques à pâte calcaire monochrome sont elles aussi toutes recouvertes d'engobe blanc, à l'exception des quatre petits vases de type $G$ qui ont été trempés dans l'engobe rouge à l'intérieur et à l'extérieur, donnant une coloration brun rouge vif.

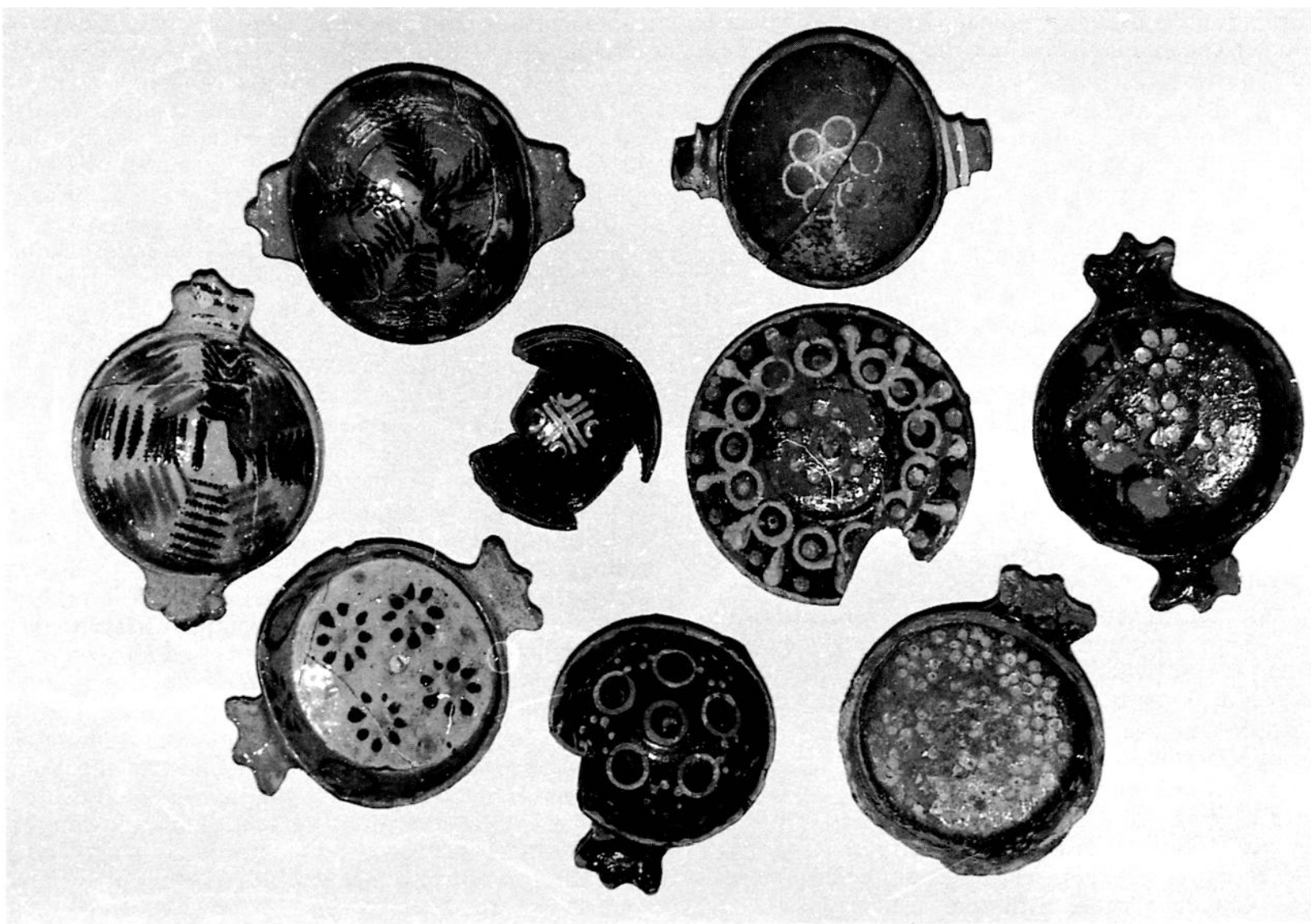

Fig. 6: Ecuelles en pâte calcaire à décor d'engobes (cl. V. Buravand). 


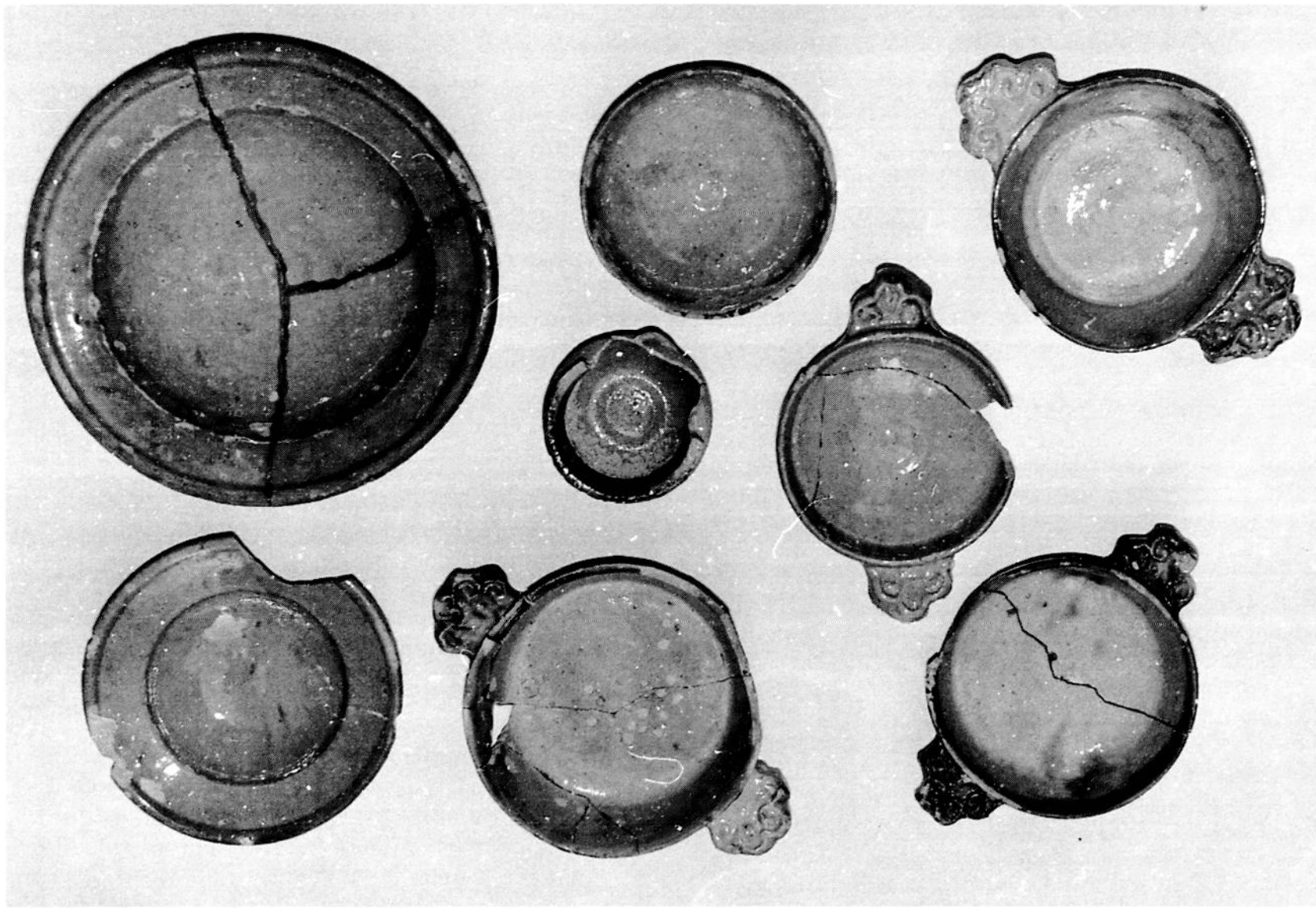

Fig. 7 : Assiettes et écuelles monochromes en pâte kaolinitique de l'Uzège (cl. V. Buravand).

Selon les pièces, les glaçures plombifères prennent des colorations jaune pâle, orangé, jaune-miel, jaune verdâtre et ces nuances résultent d'adjonction intentionnelle ou accidentelle (diffusion) de fer ou de cuivre.

Sur la totalité des céramiques monochromes jaunes, on décompte 67 écuelles de type $A 1,18$ de type $A 2$, 1 bol de type A5, 1 assiette de type B1,2 de type B2, 1 de type B5, 1 faisselle, 3 fonds de cruche et une gargoulette.

Parmi cette vaisselle jaune écrasante, figurent seulement 8 pièces vertes: 7 écuelles de type A1 et 1 assiette de type B5.

\subsubsection{Les céramiques engobées, glaçurées et} décorées

A l'intérieur de ce groupe qui représente $32 \%$ du matériel à pâte calcaire, on dénombre quatre stylcs dc décors :

2.3.2.1. Le décor de marbrures.

2.3.2.2. Le décor de cercles et points.

2.3.2.3. Le décor végétal.

2.3.2.4. Le décor géométrique.

2.3.2.1. Les céramiques marbrées représentent environ $85 \%$ des céramiques décorées (soit 60 pièces). $\mathrm{Ce}$ décor est difficilement représentable graphiquement car plus ou moins intimement mêlé (Fig. $5: 6-8$ ).

Certaines pièces présentent des marbrures d'engobe rouge sur un fond blanc et d'autres des marbrures blanches sur fond rouge.

Tout ce matériel décoré d'engobes mêlés tourbillonnés est recouvert d'un vernis transparent, taché d'oxyde de cuivre vert pour certaines des céramiques. On peut reconnaître trois techniques différentes:

- la première consiste en de larges surfaces d'engobe blanc ne se mêlant pas vraiment au fond d'engobe rouge mais semblant plutôt être obtenues de façon rapide à l'aide d'un pinceau ou d'une plume (Fig. $5: 8$. Ecuelle Type Al).

- Avec la deuxième technique, le décor est obtenu par le larges traits d'engobe, des aplats verticaux - disposés soit sur le marli pour les assiettes, soit sur les parois pour les écuelles - qui deviennent tourbillons sur le fond de l'objet, laissant supposer là aussi une exécution rapide. 12 pièces sont traitées de cette manière, donc $20 \%$ des céramiques marbrées. Une seule écuelle (A1) présente des aplats jaunes sur engobe rouge, les autres étant polychromes: les traits rouges s'intercalent alors avec les verts avant de se mêler sur le fond (Fig. 8).

Les formes concernées sont les suivantes : 8 écuelles de type A1, 2 écuelles de type A2, 1 assiette de type B2, 1 assiette de type B4.

- La troisième technique est la plus employée ( $80 \%$ des céramiques marbrées soit 48 pièces).

Dans ce cas, les aplats au pinceau n'existent pas. Les marbrures ne sont pas interrompues, elles sont au contraire continues sur les parois et le fond des écuelles et sur toute la surface des assiettes. Les tourbillons semblent former des fleurs aux multiples pétales (six pour la plupart, huit pour quelques pièces); l'effet décoratif paraît plus raffiné que celui obtenu avec la technique précédente (Fig. 9 et Fig. 10).

Cinq écuelles de type $\mathrm{A} 1$ seulement présentent ce décor foliacé sur fond rouge, et deux d'entre elles sont bichromes (engobe blanc sur engobe rouge) alors que les trois autres présentent du vert. Toutes les autres pièces sont à fond blanc, soit bichromes pour six écuelles (A1), soit polychromes pour les 37 céramiques restantes.

Plusieurs formes ont ce type de décor : 44 écuelles de type $\mathrm{A} 1 ; 1$ écuelle de type A2;1 assiette de type $\mathrm{B} 2 ; 1$ 

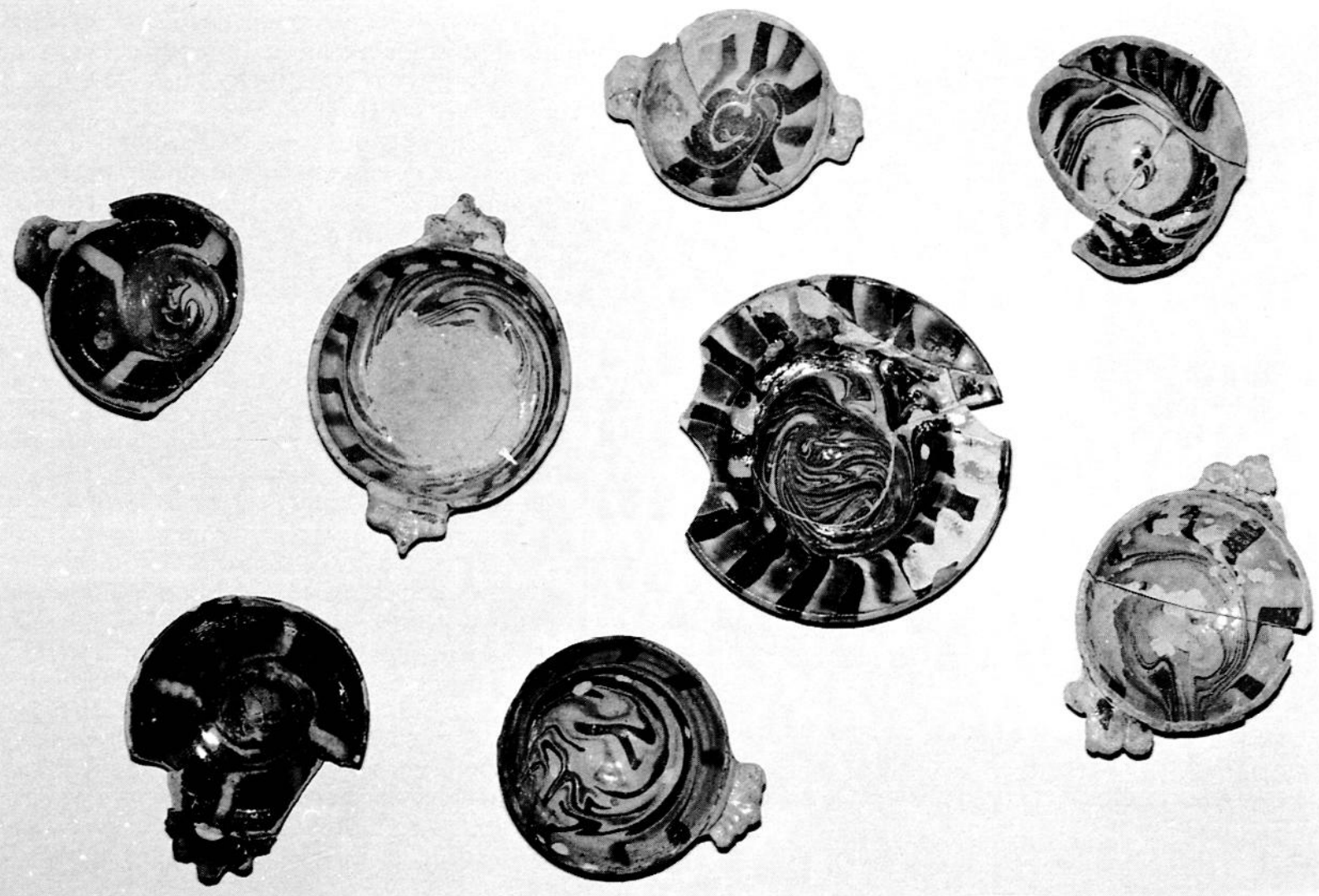

Fig. 8 : Céramiques en pâte calcaire : décor d'engobes marbrés tourbillonnant au centre (cl. V. Buravand).

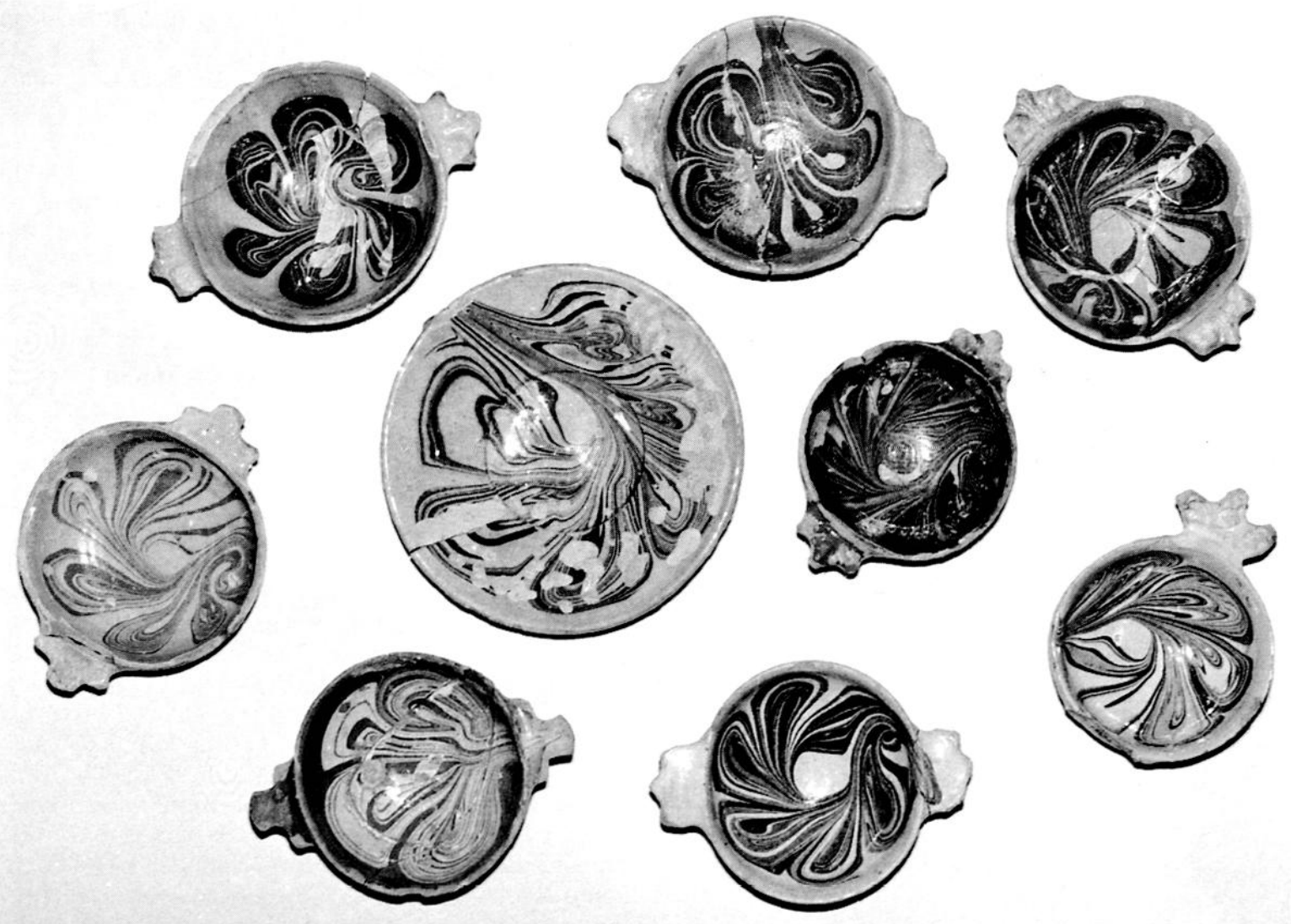

Fig. 9: Céramiques en pâte calcaire : décor d'engobes marbrés peignés (cl. V. Buravand). 

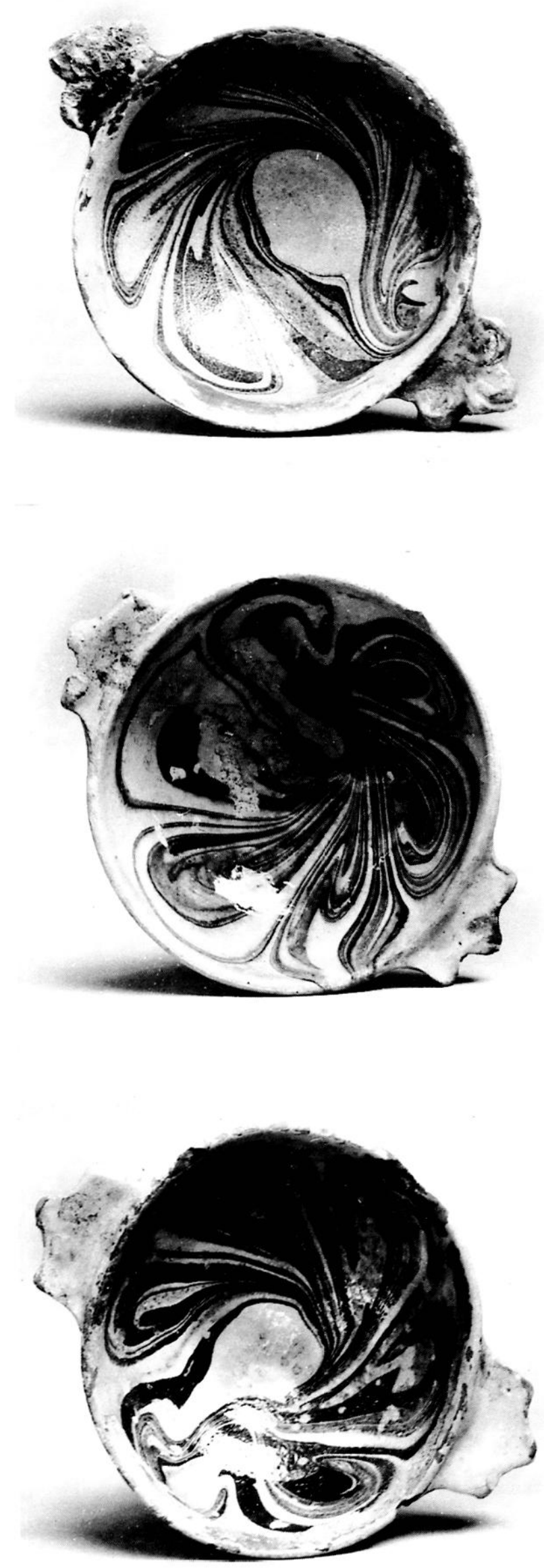

Fig. 10: Détail du décor d’engobes marbrés et peignés formant six lobes (cl. V. Buravand). assiette de type $\mathrm{B} 3 ; 1$ assiette de type B4.

2.3.2.2. Les céramiques décorées de cercles et points sont beaucoup moins nombreuses dans le lot de Boulbon que celles marbrées $(10 \%$ des céramiques décorées : 7 pièces).

Le décor obtenu à l'aide du barolet pour les points et d'un « verre de lampe " pour les cercles est traité selon trois méthodes.

- Un engobe rouge avec des cercles blancs et des points verts d'oxyde de cuivre, ou simplement des points blancs et des points verts, sous un vernis transparent, décor qui concerne une écuelle de type $\mathrm{A} 1$, deux écuelles de type A2 et une assiette de type B1 (Fig. $3: 2$ et Fig. $4: 4$ ).

- Un engobe blanc avec des cercles d'engobe rouge et toujours les taches d'oxyde de cuivre. Ce décor se retrouve sur une seule écuelle A2 (Fig. 3:6).

Le décor de points, et parfois de cercles et points, figure des fleurs dont le cœur, suggéré par un point central, est dans la majorité des cas d'une couleur différente de celle des points représentant les pétales (au nombre de 7 ou 8).

Un décor plus exceptionnel de six cercles blancs accolés autour d'un cercle central, sur fond d'engobe rouge, ne se retrouve que sur deux écuelles jumelles carénées de type Al (Fig. 3:5). Deux lignes d'engobe blanc décorent les oreilles et une souligne la lèvre. Ce motif sans réplique dans notre région est présent dans un contexte lyonnais du XVII ${ }^{\text {c }}$ siècle ( $A$ la fortune $d u$ pot, $1991: \mathrm{n}^{\circ} 252$, : 156-175; Becker 1989: fig. 25.153b, p. 166).

\subsubsection{Le décor végétal}

Deux écuelles à oreilles hémisphériques ont un décor végétal de même inspiration. Sur la première, un engobe blanc sert de fond au décor d'engobe rouge qui forme six branches stylisées aux fines ramifications obliques, toutes parallèles les unes aux autres, de part et d'autre des branches (Fig. 3:1). Ces évocations de palmes (ou encore d'arêtes de poisson) rayonnent du centre vers la périphérie.

Quant à l'autre décor, cette fois polychrome, il consiste en des «palmes» rouges et vertes s'intercalant sur un fond d'engobe blanc. La disposition du motif est toujours la même mais les "branches» sont au nombre de huit et surtout sont plus grossières, moins précises. En certains endroits, la «palme » n'est suggérée que par des traits horizontaux, parallèles les uns aux autres. Le motif est en quelque sorte plus stylisé (Fig. 5:7). Une assiette possédant ce même type de décor a été retrouvée dans un dépotoir du XVII siècle sur le site du garage Régina, rue J. Vernet à Avignon (renseignement aimablement communiqué par D. Carru).

2.3.2.4. La seule tasse présente dans le lot de Boulbon possède un décor géométrique unique qui se trouve au centre (Fig. $4: 1$ ). Ce décor, sur fond d'engobe rouge, est fait de deux traits parallèles d'engobe blanc se croisant en leur centre. Dans chacune des quatre portions d'espace créées à l'extérieur de ces lignes, un demi-cercle a été dessiné.

\section{LES VERRES}

A cet important ensemble de vaisselle s'ajoutent environ 60 fragments de verre et une pièce quasiment entière. Parmi les fragments les plus importants sont reconnaissables: 11 goulots, 3 fonds, 1 bec verseur, 1 pied, 1 anse et 1 verre de lampe; les autres appartenant sans doute à des parois d'objets, fioles ou burettes. La pièce la mieux conservée est un petit flacon de couleur bleutée dont une partie du goulot et le rebord ont disparu (Fig. 5: 11). Sa panse dont les flancs sont aplatis est 
piriforme, le fond est concave. Elle se rapproche des petites fioles présentes dans la collection de Brion (Démians d'Archimbaud 1980: 152).

Quatre goulots en verre incolore et transparent, parmi les 11 reconnaissables sont décorés sur leur partie supérieure d'un fil de verre enroulé autour d'une ouverture en forme d'entonnoir (Fig. 11-12).

- L'un, de $65 \mathrm{~mm}$ de longueur, $15 \mathrm{~mm}$ de diamètre et 35 $\mathrm{mm}$ de diamètre d'ouverture environ, possède donc ce fil de verre enroulé de couleur bleue. Le col est également bagué d'un cordon de verre bleu assez épais (Fig. 12).

- L'autre est légèrement moins long $(55 \mathrm{~mm})$, son diamètre est de $18 \mathrm{~mm}$ et son diamètre d'ouverture de 33 $\mathrm{mm}$. Le fil de verre est bleu mais enroulé de façon plus lâche. Sa particularité est de posséder une anse verticale, rattachée au haut de la panse et approximativement au milieu du col (Fig. 12).

Les autres goulots sont beaucoup plus incomplets : 4 sont en forme d'entonnoir, aux parois évasées et parmi eux, 2 en verre incolore possèdent un rebord simple, arrondi. Les 2 autres - apparemment identiques bien que l'un d'eux soit très fragmenté - sont en verre bleu devenant très épais au-dessus du niveau d'un étranglement et surtout au niveau du bord $(6 \mathrm{~mm})$ qui est plat.

Un autre goulot est également en verre bleu épais (3 $\mathrm{mm}$ ) et opaque. Il semble beaucoup moins long que les autres. En revanche, les deux derniers goulots également assez courts sont en verre bleuté très fin.

Les trois fonds sont concaves de façon plus ou moins prononcée. L'un est en verre bleu et les deux autres en verre incolore transparent. Deux ont un diamètre de $40 \mathrm{~mm}$ et un de $45 \mathrm{~mm}$. L'unique bec verseur est en verre fin incolore, il est long, fin et recourbé (Fig. 13). L'anse est en verre jaunâtre, elle ressemble à celle du goulot déjà étudié bien que de forme moins courbe (Fig. 13). Le verre de lampe est de couleur bleue, il possède un fond plat ( $20 \mathrm{~mm}$ de diamètre) et des parois évasées.

Quant au pied, de $47 \mathrm{~mm}$ de diamètre, en verre jaunâtre, il est de forme conique et les bords sont arrondis (Fig. 13). Les parois évasées de l'objet vraisemblablement impossible à identifier avec précision lui sont immédiatement rattachées, il n'existe donc pas de tige venant s'intercaler.

\section{CONCLUSION}

Au terme de cet inventaire, il apparaît que les formes et le vocabulaire décoratif des céramiques sont bien représentatifs du répertoire des vaisseliers provençaux du courant du XVII ${ }^{\mathrm{c}}$ siècle. Les productions à cette époque stéréotypées sont souvent atypiques et difficiles à attribuer à un centre producteur, en l'absence d'analyses géochimiques des pâtes. Ainsi s'il paraît possible à l'œil d'identifier la production en pâte kaolinitique de l'Uzège, il est quasiment impossible de séparer avec sécurité les productions en pâte calcaire "marseillaises" de la vallée de l'Huveaune (Abel 1987-1988) de celles du bassin proche de Saint-Maximin et de l'arrière-pays varois (Carrazé 1987) ou de celles de la région avignonnaise datées du milieu et de la seconde moitié du XVII ${ }^{\mathrm{c}}$ siècle.

Les modèles circulent ainsi que les hommes, et les techniques simples de marbrures ou de décors au barolet sont facilement reproductibles. La situation est d'autant plus complexe, lorsqu'on doit analyser des écuelles ou assiettes qui sont pour la plupart monochromes!

Cependant l'abondance et l'homogénéité de la vaisselle retrouvée permettent de supposer un approvisionnement proche, issu soit d'ateliers tarasconnais et/ou avignonnais dont la présence est bien connue dans les textes (Amouric 1986). Le décor marbré, peigné à six lobes pourrait être l'illustration d'une production locale originale.

On note toutefois que les ateliers de l'Uzège restent un centre d'approvisionnement complémentaire, même s'il n'est plus aussi spécialisé qu'au Moyen Age.

La présence d'une majolique ligure attribuée aux productions albisolaises du début du XVII ${ }^{\mathrm{e}}$ siècle, renforce la chronologie proposée par l'ensemble des céramiques régionales et des verres, et le prix-fait de l'église qui fixe un terminus aquo.

Si le problème de l'accumulation de ces vaisselles dans une église et de leur abandon reste encore sans réponse, leur présence répétée à Boulbon, comme à Notre-Dame du Bourg à Digne (Vallauri, 1989), illustre sans doute le rôle de sociabilité que fut celui de l'église au sein de la communauté villageoise.

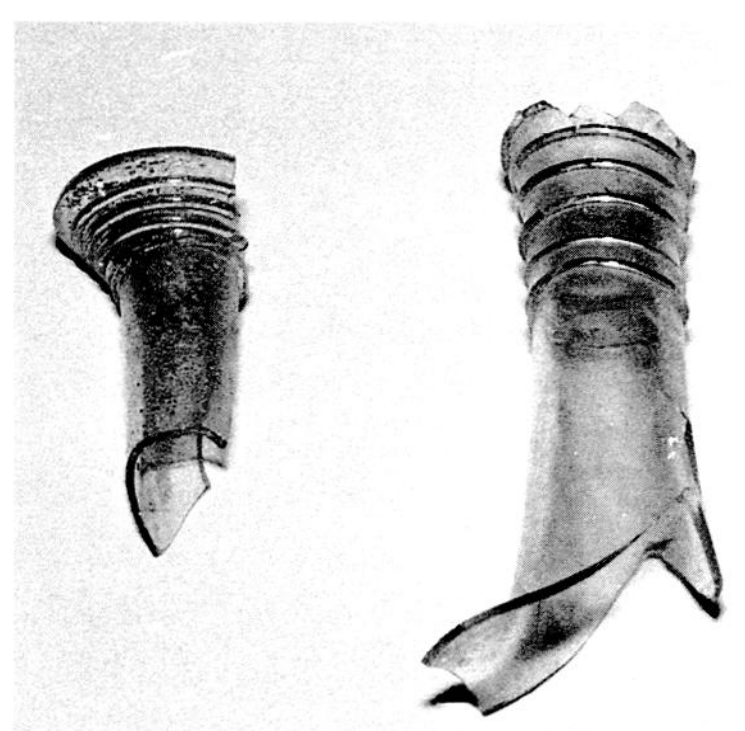

Fig. 11 : Verrerie : goulots de fioles filetés de verre blanc (cl. V. Buravand)

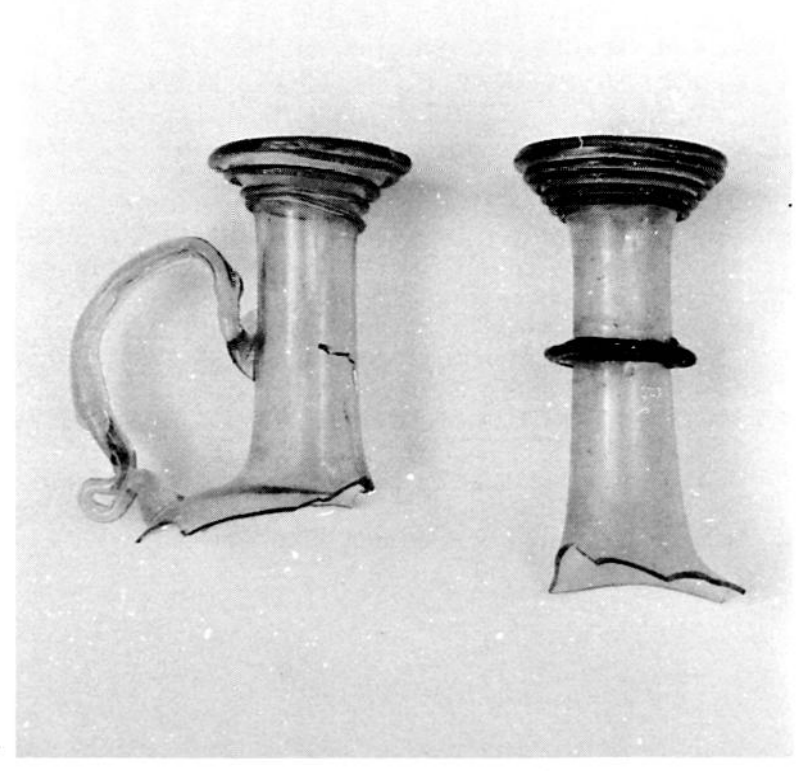

Fig. 12 : Verrerie : goulots de fioles entourés d'un fil de verre bleu (cl. V. Buravand). 


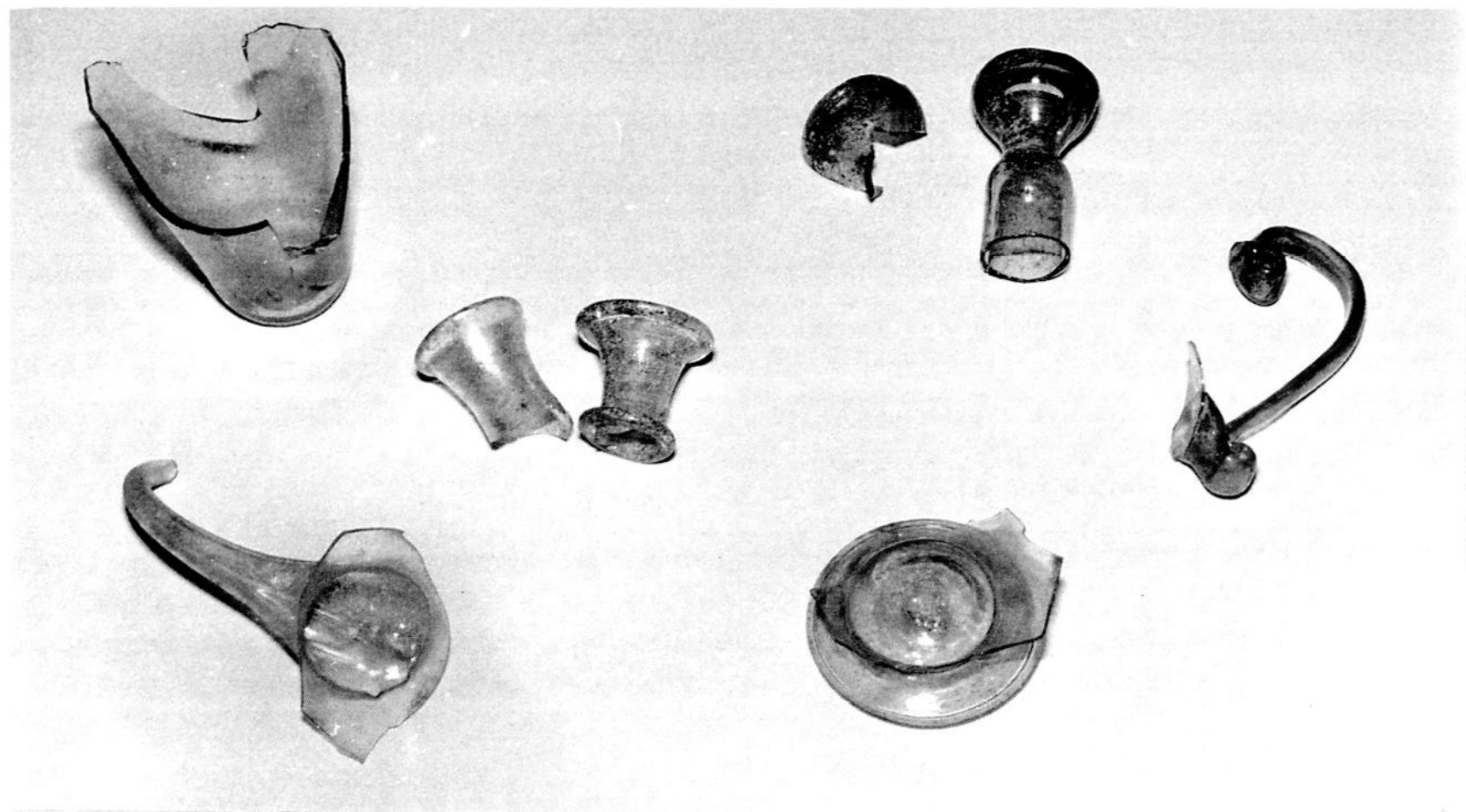

Fig. 13 : Verrerie : fond de lampe, goulots, bec verseur, anse et fond de fioles (cl. V. Buravand).

\section{BIBLIOGRAPHIE}

ABEL 1987: ABEL (V.), La céramique commune à Marseille au XVII ${ }^{e}$ siècle : l'exemple de dépotoirs domestiques du site de la Charité, Archéologie du Midi Médiéval, tome V, 1987, p. 153-165.

ABEL 1988 : ABEL(V.), La céramique du $\mathrm{XVI}^{\mathrm{e}}$ siècle sur le site de la Bourse : premiers éléments pour une typologie à Marseille (Bouches-du-Rhône), Archéologie du Midi Médiéval, Tome VI, 1988, p. 161-172.

A LA FORTUNE DU POT 1991: A la fortune du pot. La cuisine et la table à Lyon et à Vienne, $X^{e}-X I X^{e}$ siècles d'après les fouilles archéologiques, catalogue d'exposition, Lyon, Vienne, Mâcon, 1990-1991.

AMOURIC 1986: AMOURIC (H.), DEMIANS D'ARCHIMBAUD (G.), Potiers de terre en Provence Comtat Venaissin au Moyen-Age, Artistes, artisans et production artistique au Moyen Age, vol. 1, Les Hommes, Rennes, 1983-1986, p. 601-623.

BARILE 1975: BARILE (C.), Antiche ceramiche liguri, Maïoliche di Genova e Savona, Savona, 1975.

BECKER 1989 : BECKER (C.), MACE (S.), MANDY (M.-O.), VICARD (T.), AUGER (M.), Fouille de la place Antonin Poncet à Lyon, Archéologie du Midi Médiéval, tome 7, 1989 , p. $137-186$.

CARRAZE 1987: CARRAZE (F.), La poterie commune à décor baroque dans l'arrière-pays marseillais au travers des fouilles de l'ancienne Cour de Justice de Saint-Maximin, Bulletin de l'Association Polypus, St-Maximin, 1987.

CARRU 1989: CARRU (D.), Céramiques d'un dépotoir du $\mathrm{XVI}^{\mathrm{e}}$ siècle à Avignon, Archéologie du Midi Médiéval, tome 7,1989 , p. $187-210$.

DEMIANS D'ARCHIMBAUD 1980 A : DEMIANS D'ARCHIMBAUD (G ) THIRIOT (J), VALLAURI (L ) avec la collaboration de D. Foy. Céramiques d'Avignon, les fouilles de l'Hôtel de Brion et leur matériel, mémoires de l'Académie de Vaucluse, Avignon, $7^{\mathrm{C}}$ série, tome 1, 1980.
DEMIANS D'ARCHIMBAUD 1980 B : DEMIANS D'ARCHIMBAUD (G), PICON (M.), Les céramiques médiévales en France méditerranéenne. Recherches archéologiques et de laboratoire, La céramique médiévale en Méditerranée occidentale, colloque international C.N.R.S., Paris, 1980, p. 15-42.

FOY 1983 : FOY (D.), AVEROUS (J.-C.), BOURREL (B.), Peyremoutou : une verrerie du XVII ${ }^{\mathrm{e}}$ siècle dans la montagne Noire (Tarn), Archéologie du Midi Médiéval, tome 1, 1983, p. $93-102$.

FOY 1986 : FOY (D.), RICHEZ(F.), VALLAURI (L.), La céramique en usage dans l'atelier de verrier de Roquefeuille (Pourrières, Var), Archéologie du Midi Médiéval, tome IV, 1986, p. 135-149.

MARZINOT 1979: MARZINOT (M.), Ceramica e ceramisti di Liguria, Genova, 1979.

PASQUALINI 1988: PASQUALINI (M.), VALLAURI (L.), Fouilles de Toulon, Quartier de Besagne : périodes médiévales et modernes (Bouches-du-Rhône), Archéologie du Midi Médiéval, tome 6, 1988, p. 173-183.

THIRIOT 1983 : THIRIOT (J.), Aspects des terres cuites en Uzège, $X I I^{e}-X X^{e}$ siècles, Catalogue de l'exposition de SaintQuentin-La-Poterie, 1983.

THIRIOT 1985 A : THIRIOT (J.), AMOURIC (H.), VAYSETTES (J.-L.), La terre cuite en Uzège. Un artisanat ancien, catalogue de l'exposition de Saint-Quentin-La-Poterie, 1985.

THIRIOT 1985 B : THIRIOT (J.), Les ateliers de potiers postmédiévaux de Saint-Quentin-La-Poterie (Gard), état de la recherche, Archéologie du Midi Médiéval, tome 2, 1985, p. 123-150.

VALLAURI 1989: VALLAURI (L.), Découverte de lots de vaisselles d'époque moderne dans des églises, 14, NotreDame du Bourg, Digne (Alpes-de-Haute-Provence), 15, Sainte-Anne de Boulbon (Bouches-du-Rhône), L'église et son environnement, catalogue de l'exposition, Aix-en-Provence, 1989 , p. $47-49$ 